\title{
QUASICONFORMAL MAPPINGS AND AHLFORS-DAVID CURVES
}

\author{
PAUL MACMANUS
}

\begin{abstract}
We show that if $\rho$ is a quasiconformal mapping of the plane whose dilatation satisfies a certain quadratic Carleson measure condition relative to an Ahlfors-David curve $\Gamma$ then $\rho$ is differentiable almost everywhere on $\Gamma$ and $\log \left|\rho^{\prime}\right| \in$ BMO. When $\Gamma$ is chord-arc we show that its image is a BishopJones curve. If the Carleson norm is small then we show that $\rho$ is absolutely continuous on $\Gamma$, the image of $\Gamma$ is an Ahlfors-David curve, and $\rho^{\prime}=e^{a}$, where $a \in \mathrm{BMO}$ with a small norm.
\end{abstract}

\section{INTRODUCTION}

In this section results are labeled with the same numbers that they have in the text.

In general the restriction of a global quasiconformal mapping to $\mathbf{R}$ does not satisfy any regularity conditions, e.g., local absolute continuity or differentiability a.e., even when the dilatation has small $L^{\infty}$ norm. One can get some regularity by imposing some stronger smallness condition on the dilatation $\mu$. If $\mu$ were zero in a neighbourhood of $\mathbf{R}$ then the map would be smooth on $\mathbf{R}$, so if we make $\mu$ decay to zero in some sense as it approaches $\mathbf{R}$, then we should be able to get some good behaviour of the mapping on $\mathbf{R}$.

The first attempt along these lines was due to Carleson [C]. He showed that if $\rho$ is a quasiconformal map that fixes $\infty$ and maps the upper half-plane onto itself, and if its dilatation $\mu$ satisfies $|\mu(z)| \leq \varepsilon(y)$ where $\varepsilon$ is a continuous, decreasing function with $\varepsilon(0)=0$ and $\int_{0}^{1}|\varepsilon(y)|^{2} y^{-1}<\infty$, then the restriction of $\rho$ to $\mathbf{R}$ is locally absolutely continuous with a derivative in $L_{\text {loc }}^{2}$. This result was extended by Semmes [S1]. Before stating his theorem we need to make a number of definitions. We say that a measure $\lambda$ is a Carleson measure relative to a set $E$ if

$$
\sup _{\substack{x \in E \\ R>0}} \frac{1}{R} \lambda(B(x, R))<\infty .
$$

This supremum is denoted by $\|\lambda\|_{C M}$ and is called the Carleson norm of $\lambda$. Let $d_{E}(z)$ be the distance from $z$ to $E$. If $\mu$ is measurable, we define $\|\mu\|_{C}$

Received by the editors September 29, 1992 and, in revised form, February 11, 1993.

1991 Mathematics Subject Classification. Primary 30C62. BMO.

Key words and phrases. Quasiconformal mapping, Ahlfors-David curve, Carleson measures, 
relative to $E$ by

$$
\|\mu\|_{C}=\left\|\frac{|\mu(z)|^{2}}{d_{E}(z)} d x d y\right\|_{C M}
$$

where the Carleson norm on the right-hand side is taken relative to $E$. Define $\|\mu\|_{N}$ relative to $E$ by $\|\mu\|_{N}=\|\mu\|_{L^{\infty}}+\|\mu\|_{C}^{1 / 2}$. The condition $\|\mu\|_{C}<\infty$ appears in [D] and [S2].

If $\rho$ is a $K$ quasiconformal map that fixes $\infty$ and whose dilatation $\mu$ satisfies $\|\mu\|_{C}<\infty$ relative to a set $E$, then we say that $\rho \in \Sigma_{C}(E, K)$. If we are not interested in the dependence on $K$ we just write $\Sigma_{C}(E)$.

A locally rectifiable curve is $M$ chord-arc if it is a Jordan curve possibly containing $\infty$, and for any two points on the curve the length of the shortest arc joining them is bounded by $M$ times the distance between the points.

If $\Gamma$ is a locally rectifiable curve we say that $f \in \operatorname{BMO}(\Gamma)$ if

$$
f \in L_{\mathrm{loc}}^{1}(\Gamma) \text { and } \sup _{B} \frac{\int_{B \cap \Gamma}\left|f-f_{B}\right|}{H_{1}(B \cap \Gamma)}<\infty
$$

where $H_{1}$ is one-dimensional Hausdorff measure, $f_{B}=\left(\int_{B \cap \Gamma} f\right) / H_{1}(B \cap \Gamma)$, and the sup is taken over all balls centered on $\Gamma$. The supremum above is called the BMO norm of $f$ and is denoted $\|f\|_{*}$.

Semmes showed that if $\Gamma$ is an $M$ chord-arc curve then there is a constant $\gamma$, which depends only on $M$, such that if $\rho \in \Sigma_{C}(\Gamma)$ with $\|\mu\|_{N}<\gamma$ then $\rho(\Gamma)$ is a chord-arc curve, $\left.\rho\right|_{\Gamma}$ is locally absolutely continuous, and there is a complex valued function $a \in \operatorname{BMO}(\Gamma)$ with $\|a\|_{*} \leq C(M)\|\mu\|_{N}$ and $\rho^{\prime}=e^{a}$.

This extends not only Carleson's result but also a result of Dahlberg [D]. Semmes proved this result by using estimates for a certain perturbed Cauchy integral operator which he had obtained in [S2].

A related result about conformal mappings with quasiconformal extensions was proven by Astala and Zinsmeister in [AZ]. Their proof relies on estimates for the Schwarzian of $\rho$ in $\mathbf{D}$, the unit disc. They showed that if $\rho$ is quasiconformal in $\overline{\mathbf{C}}$ and conformal in $\mathbf{D}$ and $\|\mu\|_{C}$ is finite relative to $\partial \mathbf{D}$ then $\log \rho^{\prime} \in \operatorname{BMOA}(\mathbf{D})$. A corollary of this is that $\rho(\partial \mathbf{D})$ is a Bishop-Jones curve.

Bishop-Jones curves (BJ curve) are curves that are rectifiable most of the time on all scales. They were introduced in $[\mathrm{BJ}]$ and are defined as follows:

$\Gamma$ is a BJ curve if and only if: it is the boundary of a simply connected domain $\Omega$, and for $z_{0} \in \Omega$ there is a $C$ chord-arc domain $D \subseteq \Omega$ that contains $z_{0}$ and for which $H_{1}(\partial D) \leq$ $C d\left(z_{0}, \Gamma\right)$ and $H_{1}(\Gamma \cap \partial D) \geq \delta d\left(z_{0}, \Gamma\right)$.

$D$ being a chord-arc domain means that its boundary is a chord-arc curve. The corollary above follows immediately from a result in [BJ] which says that a simply connected domain $\Omega$ has boundary a BJ curve if and only if $\log \Phi^{\prime} \in$ $\operatorname{BMOA}(\mathbf{D})$, where $\Phi$ is the Riemann map from $\mathbf{D}$ onto $\Omega$. Astala and Zinsmeister use this result in [AZ] to prove that if $\Omega$ is a quasidisc and $\Phi$ is the Riemann map from $D$ onto $\Omega$ then the following are equivalent:

(i) $\Phi$ can be extended to a global quasiconformal map whose dilatation $\mu$ satisfies $\|\mu\|_{C}<\infty$ relative to $\partial \mathbf{D}$.

(0.1) (ii) $\log \Phi^{\prime} \in \operatorname{BMOA}(\mathbf{D})$.

(iii) $\partial \Omega$ is a $B J$ curve. 
A locally rectifiable curve is a $\lambda$ Ahlfors-David curve (abbreviated to $\lambda$ A-D) if the length of curve contained in any ball centered on the curve is bounded by $\lambda$ times the radius of the ball. These curves are important because they are exactly the set of curves on which the Cauchy integral is a bounded operator from $L^{2}$ to $L^{2}$.

We will extend Semmes' theorem to A-D curves and we will also be able to describe what happens when there is no restriction on the size of $\|\mu\|_{C}$. The techniques developed will allow us to give a new proof of Astala and Zinsmeister's result and its corollary.

Theorem 6.2. If $\Gamma_{0}$ is a $\lambda$ Ahlfors-David curve and $\rho \in \Sigma_{C}\left(\Gamma_{0}, K\right)$ then

(i) $\rho$ is differentiable a.e. on $\Gamma_{0}$.

(ii) $\left|\rho^{\prime}\right|>0$ a.e., $\psi=\log \left|\rho^{\prime}\right| \in \mathrm{BMO}\left(\Gamma_{0}\right)$ and

$$
\|\psi\|_{*} \leq C(\lambda, K)\left(\|\mu\|_{C}+\|\mu\|_{C}^{1 / 2}\right) .
$$

(iii) If $\Gamma_{0}=\rho\left(\Gamma_{0}\right)=\mathbf{R}$ or $\Gamma_{0}=\rho\left(\Gamma_{0}\right)=\mathbf{T}$ then $\rho$ is locally absolutely continuous, $\left|\rho^{\prime}\right| \in A_{\infty}$ and the $A_{\infty}$ norm is bounded by $A e^{C(K)\left(\|\mu\|_{C}+\|\mu\|_{C}^{1 / 2}\right)}$.

It is worth noting that (iii) in the above theorem and a version of Semmes theorem can be deduced from results of Fefferman, Kenig, and Pipher [FKP].

The next result shows that we can get more information when $\|\mu\|_{C}$ is small.

Theorem 6.3. Let $\Gamma_{0}$ be a $\lambda$ A-D curve and $\rho \in \Sigma_{C}\left(\Gamma_{0}, K\right)$. Then there exists $\varepsilon_{0}=\varepsilon_{0}(\lambda, K)$ such that if $\|\mu\|_{C} \leq \varepsilon_{0}$ then

(i) $\rho$ is locally absolutely continuous on $\Gamma_{0}$.

(ii) $\rho$ is differentiable a.e. on $\Gamma_{0}$.

(iii) $\rho^{\prime}=e^{\phi}$ where $\phi \in \mathrm{BMO}\left(\Gamma_{0}\right)$ and $\|\phi\|_{*} \leq C(\lambda, K)\|\mu\|_{C}^{1 / 2}$.

If $\rho$ satisfies the conditions of Theorem 6.3 then as corollaries of that result we get that $\rho\left(\Gamma_{0}\right)$ is a $C(\lambda, K)$ A-D curve and that $\rho\left(\Gamma_{0}\right)$ is $C(M, K)$ chordarc if $\Gamma_{0}$ is $M$ chord-arc.

We also prove a result that says that, up to a dilation, $\rho$ is bi-Lipschitz most of the time on all scales. This result is reminiscent of a result of Jones [J] which says that any Lipschitz mapping is actually bi-Lipschitz on a set of large measure.

Theorem 6.6. Let $\rho$ and $\Gamma_{0}$ be as in Theorem 6.2. Then, given $\varepsilon>0$, there exists $M=M\left(\lambda, K, \varepsilon,\|\mu\|_{C}\right)$ such that if $B$ is any ball centered on $\Gamma_{0}$ there is a measurable set $W \subseteq B$ for which

$$
\begin{aligned}
& \frac{H_{1}\left(\left(\Gamma_{0} \cap B\right) \backslash W\right)}{H_{1}\left(\Gamma_{0} \cap B\right)} \leq \varepsilon \text { and } \\
& \left.\frac{\operatorname{diam}\left(\Gamma_{0} \cap B\right)}{\operatorname{diam}\left(\rho\left(\Gamma_{0} \cap B\right)\right)} \rho\right|_{W} \text { is } M \text { bi-Lipschitz. }
\end{aligned}
$$

This theorem allows us to describe the images of chord-arc domains as follows:

Theorem 6.7. If $D$ is an $\alpha$ chord-arc domain, $\Gamma_{0}=\partial D$ and $\rho$ is a $K$ quasiconformal map whose dilatation, $\mu$, satisifes $\|\mu\|_{C}<\infty$ relative to $\Gamma_{0}$ then $\rho\left(\Gamma_{0}\right)$ is a BJ curve with constants that depend only on $\alpha,\|\mu\|_{C}, K$. 
It should be noted that this result can also be obtained by combining various results in [AZ, FKP, and $\mathrm{S} 1]$.

Combining this with $(0.1)$ we see that a quasidisc has boundary a BJ curve if and only if it is the image of a chord-arc domain $D$ under a global quasiconformal map whose dilatation $\mu$ satisfies $\|\mu\|_{C}<\infty$ relative to $\partial D$. Alternatively, we have that a curve $\Gamma$ is a BJ curve and a quasicircle if and only if it is the image of a chord-arc curve under a quasiconformal map of the type that we are considering. Thus we have an equivalence between a set of geometric conditions on a curve and a certain type of quasiconformal mapping.

In $\S 1$ we describe a good parameterisation of our curve and in $\S 3$ we use this to cut the curve up in a controlled way at all scales. In the second part of $\S 1$ we show how we use this to reduce the problem to one of estimating Haar type coefficients for the logarithm of the derivative. In $\S 2$ we prove the main lemma, which allows us to describe the behaviour of the Haar coefficients by giving an estimate on the distortion produced by a quasiconformal map in terms of quadratic integrals involving the dilatation. As an interesting corollary we easily obtain an estimate for the circular distortion of a quasiconformal mapping which is of the right order when the quasiconformal constant is close to 1 . In $\S 4$ we describe the relation between $\mathrm{BMO}$ and estimate on coefficients and in $\S 5$ we study BMO on A-D curves and we prove a useful lemma that allows us to identify exponentials of small BMO functions. The proofs of the various theorems are in $\S 6$.

This paper is a revised version of my doctoral thesis, which was written at Yale University. I would like to take this opportunity to thank my advisor, Peter W. Jones, without whose guidance and enthusiasm this project would never have been completed.

\section{Preliminaries}

A set $\Gamma$ in the plane is called a locally rectifiable curve if $\Gamma$ is closed, connected, and $H_{1}(\Gamma \cap B)<\infty$, where $B$ is any ball in the plane and $H_{1}$ is one-dimensional Hausdorff measure (see $[\mathrm{F}]$ for the definition of $H_{1}$ ).

A locally rectifiable curve $\Gamma$ is rectifiable if $H_{1}(\Gamma)<\infty$. Such a curve is compact and is also referred to as a rectifiable continuum. A rectifiable continuum is arc connected (see $[F]$ ).

A useful fact about locally rectifiable curves is that any bounded subset of such a curve is contained in a rectifiable subcurve of the original curve. One consequence of this is that any locally rectifiable curve is arc connected.

We normally think of a curve as having some parameterisation. The next proposition justifies calling the sets described above curves. Before stating it we need a definition. A map $z$ from an interval $I_{0}$ to $\Gamma$ will be called an $M$-parameterisation if it satisfies

(i) $z$ is continuous and onto.

(ii) $\operatorname{diam}(\Gamma) \leq M|z(a)-z(b)|$, where $a, b$ are the endpoints of $I_{0}$.

(iii) If $E$ is any Borel set in $I_{0}$ then

$$
H_{1}(z(E)) / M \leq|E| \leq M H_{1}(z(E)) \text {. }
$$

From (iii) we see that $z$ is an $M$-Lipschitz mapping and that $1 / M \leq\left|z^{\prime}\right| \leq M$. We can also deduce from (iii) that, except for a set of measure zero, there is a 
uniform bound on the number of times that the parameterisation can hit any given point.

Proposition 1.1. If $\Gamma$ is a rectifiable curve then there is a 2-parameterisation of $\Gamma$.

Outline of proof. From Lemma 3.13 in $[\mathrm{F}]$ we know that $\Gamma$ can written as a countable union of rectifiable Jordan curves $\left\{\Gamma_{j}\right\}$, and a set of measure zero. Furthermore, the distance between the endpoints of $\Gamma_{1}$ is equal to the diameter of $\Gamma$ and $\Gamma_{n+1} \cap\left(\bigcup_{j=1}^{n} \Gamma_{j}\right)$ is exactly one of the endpoints of $\Gamma_{n+1}$. Let $C_{n}=$ $\bigcup_{j=1}^{n} \Gamma_{j}$. It is easy to find a 2-parameterisation of each $C_{n}$ and the proposition follows by taking a limit of these parameterisations.

We will refer to this as an arclength parameterisation of $\Gamma$. This parameterisation will be very useful later on because it will allow us to transform the study of functions on a curve to the study of functions on an interval. The next proposition will allow us to transfer results from the interval back to the curve. It should be noted that in the case where $\Gamma$ is actually a Jordan curve the parameterisation given above is a little bit different from the usual arclength parameterisation of a rectifiable Jordan curve (see [F] for the usual parameterisation). This is because of condition (ii) in the definition of an $M$-parameterisation.

If $f$ is defined on a locally rectifiable curve $\Gamma$ we say that $f$ is differentiable at $x \in \Gamma$ if there is a number $A$ such that

$$
f(y)=f(x)+A(y-x)+o(|y-x|)
$$

for $y \in \Gamma . A$ is called the derivative of $f$ at $x$ and is denoted by $f^{\prime}(x)$.

We define $f$ to be absolutely continuous on $\Gamma$, a rectifiable curve, if $\forall \varepsilon>$ $0 \exists \delta>0$ such that if $\left\{E_{n}\right\}_{n=1}^{\infty}$ is a collection of disjoint connected subsets of $\Gamma$ with $\sum_{n=1}^{\infty} H_{1}\left(\Gamma \cap E_{n}\right) \leq \delta$ then $\sum_{n=1}^{\infty} \operatorname{diam}\left(f\left(\Gamma \cap E_{n}\right)\right) \leq \varepsilon$. If $\Gamma$ is locally rectifiable we say that $f$ is locally absolutely continuous if it is absolutely continuous on any rectifiable subcurve of $\Gamma$.

Proposition 1.2. If $\Gamma$ is a rectifiable curve, $z$ is an M-parameterisation of $\Gamma$, and $f$ is a function on $\Gamma$ then

(i) $f$ is differentiable a.e. on $\Gamma \Leftrightarrow f \circ z$ is differentiable a.e.

(ii) $f$ is absolutely continuous $\Leftrightarrow f \circ z$ is absolutely continuous.

We will also make use of the following

Proposition 1.3. If $\Gamma$ is a locally rectifiable curve and $f$ is locally absolutely continuous on $\Gamma$ then

(i) $f$ is differentiable a.e. on $\Gamma$ and $f^{\prime} \in L_{\mathrm{loc}}^{1}(\Gamma)$.

(ii) If $E$ is any measurable subset of $\Gamma$ then $H_{1}(f(E)) \leq \int_{E}\left|f^{\prime}\right|$. If $f$ is also one-to-one then $H_{1}(f(E))=\int_{E}\left|f^{\prime}\right|$.

Outline of proof of Proposition 1.2(i). The "only if" direction is clear.

Now for the "if" direction. Recall that a point on $\Gamma$ is said to be regular if the upper and lower (circular) densities are both one at that point. Almost every point on $\Gamma$ is a regular point (see [F]). Also, both $z$ and $f \circ z$ are differentiable a.e. Because $z$ preserves null sets we get that almost every point on $\Gamma$ is regular and has the property that both $z$ and $f \circ z$ are differentiable at all points of its preimage. Now let $x$ be one such point. 
If $f$ is differentiable at $x$ then $f^{\prime}(x)=(f \circ z)^{\prime}(a) / z^{\prime}(a)$ where $a$ is an element of $f^{-1}(x)$. To show that $f$ is differentiable at $x$ it suffices to show that for $a, b \in f^{-1}(x)$

$$
(f \circ z)^{\prime}(a) / z^{\prime}(a)=(f \circ z)^{\prime}(b) / z^{\prime}(b) .
$$

Let $I, J$ be intervals centered at $a$ and $b$ respectively. It follows from the facts that $x$ is regular and that $z$ is differentiable at $a$ and $b$, that $z(I)$ and $z(J)$ intersect in a set of positive measure, and so they have a common point other than $x$. By letting $I$ and $J$ shrink to length zero we can find a sequence of points converging to $a$ whose images are not $x$ and a similar sequence converging to $b$ such that $z$ maps the corresponding terms of the sequences to the same point. It follows immediately from this that (1.1) is true.

The proofs of the remaining parts of the two propositions are fairly straightforward so we omit them.

A-D curves were defined in the introduction. The next proposition essentially follows from the connectivity of the curve.

Proposition 1.4. $\exists N=N(\lambda)$ such that if $\Gamma$ is a $\lambda A-D$ curve and $B$ is any ball centered on $\Gamma$ then

(i) $\Gamma \cap B \subseteq \bigcup_{n=1}^{N} \gamma_{n}$, where the $\gamma_{n}$ are disjoint $\lambda A$-D curves contained in $\Gamma \cap 2 B$.

(ii) $1 / 2 \operatorname{diam}(\Gamma \cap B) \leq \operatorname{diam}\left(\gamma_{n}\right) \leq 4 \operatorname{diam}(\Gamma \cap B)$ for any $n$.

A collection $\Lambda$ of nested families of subintervals of an interval $I_{0}$ is called an $\alpha$-subdivision of $I_{0}$ if $\Lambda=\bigcup_{N=0}^{\infty} \Lambda_{N}$, where the family $\Lambda_{0}$ just consists of $I_{0}$ and the intervals of the family $\Lambda_{N+1}$ are obtained by dividing each element, $I$, of $\Lambda_{N}$ into two intervals $A, B$ so that $\alpha \geq|I| /|A|,|I| /|B| \geq 1$. For $I \in \Lambda_{N}$, $N \geq 1$, we denote by $I_{P}$ the unique element of $\Lambda_{N-1}$ that contains $I$ and for $I \in \Lambda$ we let $I_{L}, I_{R}$ be the two pieces that is it split into: $I_{L}$ being the left one and $I_{R}$ being the right one.

If $J$ is an interval, we define $|z(J)|$ to be $|z(a)-z(b)|$ where $a, b$ are the endpoints of $J$, and if $\Lambda$ is a subdivision of $I_{0}$ we say that it gives a $\beta$-subdivision of $\Gamma$ if

$$
\frac{1}{\beta} \leq \frac{\left|z\left(I_{L}\right)\right|}{|z(I)|}, \frac{\left|z\left(I_{R}\right)\right|}{|z(I)|} \leq \beta \quad \text { for any } I \in \Lambda .
$$

For $I=[a, b] \in \Lambda$, we set $x_{I}=z(a)$ and $y_{I}=z(b)$. We say that $f \in$ $\operatorname{BMO}(\Lambda)$ if

$$
f \in L_{\mathrm{loc}}^{1} \quad \text { and } \sup _{I} \frac{\int_{I}\left|f-f_{I}\right|}{|I|}<\infty,
$$

where $f_{I}$ is the mean value of $f$ on $I$ and the sup is taken over all elements of $\Lambda$. The supremum is called the BMO norm of $f$ and is denoted $\|f\|_{*}$. $\operatorname{BMO}(\Lambda)$ has many of the properties of ordinary BMO including the following version of the John-Nirenberg Theorem: If $\Lambda$ is an $\alpha$-subdivision, $f \in \mathrm{BMO}(\Lambda)$, and $I \in \Lambda$ then there exists $C(\alpha), b(\alpha)$ such that

$$
|x \in I:| g(x)-g_{I}|>t| \leq|I| C(\alpha) \exp \left(-b(\alpha) t /\|g\|_{*}\right) .
$$

The next proposition follows immediately from the above estimate. 
Proposition 1.5. Let $\Lambda$ be an $\alpha$-subdivision of $I_{0}$. There exists $\delta=\delta(\alpha)$ such that if $g \in \mathrm{BMO}(\Lambda)$ and $\|g\|_{*} \leq \delta$ then

(i) $e^{g} \in L^{1}$ and for any $I \in \Lambda$,

$$
\frac{\left|e^{g_{I}}\right|}{C(\alpha)} \leq \frac{\int_{I}\left|e^{g}\right|}{|I|} \leq C(\alpha)\left|e^{g_{I}}\right| .
$$

(ii) $\left(\int_{I}\left|e^{g-g_{I}}-1\right|\right) /|I| \leq C(\alpha)\|g\|_{*}$.

Let $\rho \in \Sigma_{C}(\Gamma), \Gamma$ be a compact $\lambda$ A-D curve and $z: I_{0} \rightarrow \Gamma$ be an arclength parameterisation of $\Gamma$. In $\S 3$ we will construct a $C(\lambda)$-subdivision $\Lambda$ of $I_{0}$ that gives a 4-subdivision of $\Gamma$. Let $E_{\Lambda}=I_{0} \backslash$ \{endpoints of the elements of $\Lambda$ \} and for $x \in E_{\Lambda}$ let $I_{N}(x)$ be the unique element of $\Lambda_{N}$ that contains $x$. We want to know that $\rho^{\prime}$ exists a.e. and we want to describe its behaviour so we define

$$
Q_{\rho}(I)=\frac{\rho(z(b))-\rho(z(a))}{z(b)-z(a)}
$$

for $I=[a, b] \in \Lambda$. If $\rho^{\prime}$ exists a.e. then $Q_{\rho}\left(I_{N}(x)\right) \rightarrow \rho^{\prime}(z(x))$ a.e. Conversely if we can get suitable bounds on $Q_{\rho}(I), \forall I \in \Lambda$ then we will be able to deduce that $\rho$ is differentiable a.e. on $\Gamma$, and we will be able to describe the behaviour of the derivative. Let

$$
F_{N}(x)=Q_{\rho}\left(I_{0}\right) \prod_{j=1}^{N} \frac{Q_{\rho}\left(I_{j}(x)\right)}{Q_{\rho}\left(I_{j-1}(x)\right)}, \quad N \geq 1,
$$

for $x \in E_{\Lambda}$ and zero otherwise. Then $F_{N}(x)=Q_{\rho}\left(I_{N}(x)\right)$, so $F_{N}(x)$ converges to $\rho^{\prime}(z(x))$ a.e. if $\rho^{\prime}$ exists a.e. The main lemma will say that for any $I \in \Lambda$ we have

$$
Q_{\rho}(I) / Q_{\rho}\left(I_{P}\right)=e^{\lambda_{I}}
$$

with an estimate on $\lambda_{I}$ in terms of some integral involving $\mu$. If $\rho$ is a Euclidean motion in some neighbourhood of $I_{P}$ then $\lambda_{I}$ will be zero, so $\lambda_{I}$ is a measure of the deviation of $\rho$ from a Euclidean motion in a neighbourhood of $I_{P}$. We can now write

$$
F_{N}(x)=Q_{\rho}\left(I_{0}\right) \exp \left\{\sum_{j=1}^{N} \lambda_{I_{j}(x)}\right\} \text { a.e. }
$$

and if we define

$$
g^{I}(x)=\lambda_{I_{L}} \chi_{I_{L}}(x)+\lambda_{I_{R}} \chi_{I_{R}}(x) \text { and } f_{N}(x)=\sum_{j=0}^{N-1} \sum_{I \in \Lambda_{j}} g^{I}(x)
$$

then we can write

$$
F_{N}(x)=Q_{\rho}\left(I_{0}\right) \exp \left\{f_{N}(x)\right\} \text { a.e. }
$$

The $g^{I}$ have some orthogonality properties. To show this we will assume for the moment that $\Gamma$ is the unit interval, thus $z$ is the identity. It is easily checked that

$$
\left|I_{L}\right|\left(e^{\lambda_{I_{L}}}-1\right)+\left|I_{R}\right|\left(e^{\lambda_{I_{R}}}-1\right)=0
$$


and because $\lambda_{I} \sim e^{\lambda_{I}}-1$ we get that $\left|I_{L}\right| \lambda_{I_{L}}+\left|I_{R}\right| \lambda_{I_{R}} \sim 0$. Thus $g I(x) \sim$ $\lambda_{I_{L}} h_{I}(x)$, where

$$
h_{I}(x)=\chi_{I_{L}}(x)-\frac{\left|I_{L}\right|}{\left|I_{R}\right|} \chi_{I_{R}}(x) \text {. }
$$

Note that the $h_{I}$ are an orthogonal family. In the general case we will also get that $g^{I}(x) \sim \lambda_{I_{L}} h_{I}(x)$. Thus

$$
f_{N}(x) \sim \sum_{j=0}^{N-1} \sum_{I \in \Lambda_{j}} \lambda_{I_{L}} h_{I}(x) \text { a.e. }
$$

The estimates of the main lemma and the geometric properties of A-D curves will show that

$$
\sup _{I \in \Lambda} \frac{\sum_{J \subseteq I}|J|\left|\lambda_{J_{L}}\right|^{2}}{|I|}<\infty
$$

This Carleson measure type condition reflects the Carleson condition on $\mu$. From equation (1.3) we can show that $f_{N} \in \mathrm{BMO}(\Lambda)$. We will also show that the $f_{N}$ converge both in $L^{1}$ and a.e. to a limit function that is also in $\operatorname{BMO}(\Lambda)$. We will denote the limit function by $f$ or by $\sum_{I \in \Lambda} \lambda_{I} h_{I}$. Once we have this, we will be able to deduce that $\rho^{\prime}$ exists a.e. and

$$
\rho^{\prime}(z(x))=Q_{\rho}\left(I_{0}\right) \exp \{f(x)\} \quad \text { a.e. }
$$

\section{The Main Lemma}

The following property of quasiconformal mappings is well known (see [LV], for example).

Proposition 2.1. If $F$ is a $K$ quasiconformal mapping of $\overline{\mathbf{C}}$ that fixes 0,1 , and $\infty$ then $\exists C(K)$ such that

$$
|z-w|^{K} / C(K) \leq|F(z)-F(w)| \leq C(K)|z-w|^{1 / K} \text { for }|z|,|w| \leq 1 .
$$

Corollary 2.2. If $F$ is as above and $R \geq 1$ then $\exists c(K)$ such that

$$
\begin{aligned}
|z|^{1 / K} / c(K) & \leq|F(z)| \leq c(K)|z|^{K} \quad \text { for }|z| \geq 1, \\
|z-w|^{K} / c(K) R^{K} \leq|F(z)-F(w)| & \leq c(K) R^{K}|z-w|^{1 / K} \text { for }|z|,|w| \leq R .
\end{aligned}
$$

Before proving the Main Lemma we will need a preliminary lemma about the size of the derivative of a quasiconformal mapping.

Lemma 2.3. If $\rho$ is a $K$ quasiconformal mapping that fixes 0,1 , and $\infty$ then there exists $p=p(K)>2$ and $h \in L^{p}$ such that

$|\partial \rho| \leq C(K)(1+|h|)$ a.e. in $B(0,6) \quad$ and $\|h\|_{p} \leq C(K)\left(\iint_{B}|\mu|^{p}\right)^{1 / p}$,

where $\mu$ is the dilatation of $\rho$ and $B=B(0,8)$.

Proof. Let $\mu_{1}=\mu \chi_{B}$ and let $\rho_{1}$ be the quasiconformal map with dilatation $\mu_{1}$ that fixes 0,1 and $\infty$. Then $\rho$ can be written as $\rho_{2} \circ \rho_{1}$, where $\rho_{2}$ is a $K$-quasiconformal map that fixes 0,1 and $\infty$ and which is conformal in $\rho_{1}(B)$. Then $\partial \rho(z)=\rho_{2}^{\prime}\left(\rho_{1}(z)\right) \partial \rho_{1}(z)$ in $B$. However, from the Koebe $1 / 4$ 
theorem and the proposition above we get that $\left|\rho_{2}^{\prime}\right| \leq C(K)$ on $\rho_{1}(B(0,6))$ and consequently $|\partial \rho| \leq C(K)\left|\partial \rho_{1}\right|$ in $B(0,6)$.

From [A] we know that there is a quasiconformal map $g$ with dilatation $\mu_{1}$ that fixes 0 and $\infty$ and for which $\partial g=1+h$ a.e., where $\|h\|_{p} \leq C(K)\left\|\mu_{1}\right\|_{p}$ for some $p>2$. Then $\rho_{1}$ must be $g / g(1)$. From [A] we also get that $|g(1)| \geq$ $c(K)$ and so we are done.

Lemma 2.4 (The Main Lemma). If $\rho$ is a $K$ q.c. map that fixes 0,1 and $\infty$ and has dilatation $\mu$, and if $z \in B(0,5) \backslash B\left(0, \frac{1}{4}\right)$, then $\rho(z) / z=e^{a}$ and

$$
|a|^{2} \leq C(K)\left\{\iint_{\mathbf{C} \backslash B} \frac{|\mu|^{2}}{|w|^{3}}+\iint_{B}|\mu|^{2}\left(\frac{1}{|w|^{1 / 2}}+\frac{1}{|w-z|^{1 / 2}}+\frac{1}{|w-1|^{1 / 2}}\right)\right\}
$$

where $B=B(0,8)$.

We also have the following uniform bound for $a$ :

$$
|a| \leq A(K)\|\mu\|_{\infty} .
$$

Proof. By choosing a sequence of smooth dilatations which are all zero in a neighbourhood of 0 and a neighbourhood of $\infty$, and which converge to $\mu$ a.e., we see that there is no loss of generality if we assume that $\mu$ has this form. Thus we can assume that $\rho$ is smooth and that it is conformal in a neighbourhood of both 0 and $\infty$.

Let $G(z)=\rho(z) / z$. Because $\rho$ is conformal in a neighbourhood of both 0 and $\infty$ and $\rho$ fixes 0 and $\infty$, we get that $G$ is conformal, bounded, and nonzero near 0 and $\infty$. Thus $G$ is smooth and nonzero in $\mathbf{C}$. The next step is to define a logarithm of $G$.

If $w \neq 0$, then we can define an analytic logarithm in some neighbourhood of $w$. Combining this with the monodromy theorem we find that we can define a smooth logarithm $L$ of $G$ such that $L(1)=0$. Clearly we have

$$
\bar{\partial} L(z)=\bar{\partial} G(z) / G(z)=\mu \partial \rho(z) / \rho(z) .
$$

Define

$$
H(z)=\frac{1}{\pi} \iint \frac{\bar{\partial} L(w)}{z-w} .
$$

Then $\bar{\partial}(L-H)=0$. But $L-H$ is continuous, so by Weyl's lemma $L-H$ is actually analytic in $\mathbf{C}$. Because $\rho$ is conformal in a neighbourhood of $\infty$ we have that $\lim _{z \rightarrow \infty} L(z)<\infty$. It is easy to check that $\lim _{z \rightarrow \infty} H(z)$ is zero, hence $\lim _{z \rightarrow \infty} L-H$ is finite. Because $L-H$ is also entire it must be constant. We can evaluate the constant by noting that $L(1)=0$, thus

$$
\begin{aligned}
|L(z)| & =\left|\frac{1}{\pi} \iint \frac{\mu \partial \rho(w)}{\rho(w)}\left(\frac{1}{z-w}-\frac{1}{1-w}\right)\right| \\
& \leq C \frac{1}{\pi} \iint \frac{|\mu||\partial \rho(w)|}{|\rho(w)|}\left(\frac{\chi_{B(z, 1 / 8)}}{|w-z|}+\chi_{B}+\frac{\chi_{B(1,1 / 8)}}{|w-1|}+\frac{\chi_{\complement \backslash B}}{|w|^{2}}\right) .
\end{aligned}
$$

We want to get an estimate on the size of each of the four terms. Let $B_{1}=$ 


$$
\begin{aligned}
& B(z, 1 / 8) \text {. } \\
& \iint \frac{|\mu||\partial \rho(w)|}{|\rho(w)|} \frac{\chi_{B(z, 1 / 8)}}{|w-z|} \leq C(K) \iint_{B_{1}} \frac{|\mu||\partial \rho(w)|}{|w-z|} \\
& \leq C(K)\left(\left(\iint_{B_{1}} \frac{|\mu|^{2}}{|w-z|^{1 / 2}}\right)^{1 / 2}+\iint_{B_{1}} \frac{|\mu||h|}{|w-z|}\right) .
\end{aligned}
$$

The first inequality is a consequence of the quasiconformal distortion estimates while the second follows from Lemma 2.3 and an application of Hölder's inequality. We know that $h \in L^{p}$ for some $p>2$. Applying Hölder's inequality with $1 / r=1 / 2-1 / p$, we get

$$
\begin{aligned}
& \iint_{B_{1}} \frac{|\mu||h|}{|w-z|} \\
& \quad \leq\left(\iint_{B_{1}} \frac{|\mu|^{r}}{|w-z|^{1 / 2}}\right)^{1 / r}\left(\iint_{B_{1}}|h|^{p}\right)^{1 / p}\left(\iint_{B_{1}} \frac{1}{|w-z|^{3 / 2+1 / p}}\right)^{1 / 2}
\end{aligned}
$$

Because $3 / 2+1 / p<2$, the last term in the product is bounded by some constant depending only on $p$ and hence on $K$. Using Lemma 2.3 on the second term, we get

$$
\begin{aligned}
\iint_{B_{1}} \frac{|\mu||h|}{|w-z|} & \leq C(K)\left(\iint_{B} \frac{|\mu|^{r}}{|w-z|^{1 / 2}}\right)^{1 / r}\left(\iint_{B}|\mu|^{p}\right)^{1 / p} \\
& \leq C(K)\left(\iint_{B} \frac{|\mu|^{2}}{|w-z|^{1 / 2}}\right)^{1 / r}\left(\iint_{B} \frac{|\mu|^{2}}{|w-z|^{1 / 2}}\right)^{1 / p} \\
& \leq C(K)\left(\iint_{B} \frac{|\mu|^{2}}{|w-z|^{1 / 2}}\right)^{1 / 2}
\end{aligned}
$$

Thus the first term in (2.4) is bounded by

$$
C(K)\left(\iint_{B} \frac{|\mu|^{2}}{|w-z|^{1 / 2}}\right)^{1 / 2} \text {. }
$$

Similarly, the third term is bounded by

$$
C(K)\left(\iint_{B} \frac{|\mu|^{2}}{|w-1|^{1 / 2}}\right)^{1 / 2} .
$$

We will deal with the second term next.

$$
\iint \frac{|\mu||\partial \rho|}{|\rho|} \chi_{B} \leq\left(\iint_{B} \frac{|\mu|^{2}}{|w|^{1 / 2}}\right)^{1 / 2}\left(\iint_{B} \frac{|\partial \rho|^{2}|w|^{1 / 2}}{|\rho|^{2}}\right)^{1 / 2} .
$$

Let $A_{n}=\left\{w: 2^{-n-1}<|w| \leq 2^{-n}\right\}$. From the distortion estimates for quasiconformal maps we get that

$$
\frac{1}{C(K)} \leq \frac{\text { Area of } \rho\left(A_{n}\right)}{|\rho(z)|^{2}} \leq C(K), \quad \text { for any } z \in A_{n} .
$$

From this we get that $|\rho(z)| \sim|\rho(w)|$ where $z, w$ are any two points in $A_{n}$. The Jacobian $J$ of $\rho$ is $|\partial \rho|^{2}-|\bar{\partial} \rho|^{2}$ and because $\rho$ is quasiconformal we 
get that $J$ is positive and is bounded below by $C(K)^{-1}|\partial \rho|^{2}$. Thus if $z_{n} \in A_{n}$ we can write

$$
\begin{aligned}
\iint_{B} \frac{|\partial \rho|^{2}|w|^{1 / 2}}{|\rho|^{2}} & \leq \sum_{n \geq-4} 2^{-n / 2} \iint_{A_{n}} \frac{|\partial \rho|^{2}}{|\rho|^{2}} \\
& \leq C(K) \sum_{n \geq-4} 2^{-n / 2} \frac{\text { Area of } \rho\left(A_{n}\right)}{\left|\rho\left(z_{n}\right)\right|^{2}} \leq C(K) .
\end{aligned}
$$

Thus the second term is bounded by

$$
\left(C(K) \iint_{B} \frac{|\mu|^{2}}{|w|^{1 / 2}}\right)^{1 / 2} .
$$

All that remains is to estimate the fourth term. Let $R_{n}=\left\{w: 2^{n} \leq|w|<2^{n+1}\right\}$. As above we get that

$$
\iint_{R_{n}} \frac{|\partial \rho|^{2}}{|\rho|^{2}} \leq C(K),
$$

therefore

$$
\begin{aligned}
\iint_{\mathbf{C} \backslash B} \frac{|\mu||\partial \rho|}{|w|^{2}|\rho|} & \leq\left(\iint_{\mathbf{C} \backslash B} \frac{|\mu|^{2}}{|w|^{3}}\right)^{1 / 2}\left(\iint_{\mathbf{C} \backslash B} \frac{|\partial \rho|^{2}}{|\rho|^{2}|w|}\right)^{1 / 2} \\
& \leq\left(\iint_{\mathbf{C} \backslash B} \frac{|\mu|^{2}}{|w|^{3}}\right)^{1 / 2}\left(\sum_{n \geq 3} 2^{-n} \iint_{R_{n}} \frac{|\partial \rho|^{2}}{|\rho|^{2}}\right)^{1 / 2} \\
& \leq C(K)\left(\iint_{\mathbf{C} \backslash B} \frac{|\mu|^{2}}{|w|^{3}}\right)^{1 / 2} .
\end{aligned}
$$

Because $w^{-3}$ is integrable over $\mathbf{C} \backslash B$ and $w^{-1 / 2}$ is integrable over $B$ we obtain the uniform bound for $a$.

For $f$ a $K$-quasiconformal map of the plane that fixes $\infty$, let $L_{f}(x, f)=$ $\max _{y \in B(x, r)}|f(y)-f(x)|$ and $l_{f}(x, r)=\min _{y \in B(x, r)}|f(y)-f(x)|$. Define

$$
M(f)=\sup _{x \in \mathbf{C}} \sup _{r>0} \frac{L_{f}(x, r)}{l_{f}(x, r)} .
$$

It is an important theorem that $M(f)$ can be uniformly bounded by a constant that depends only on $K$ and that good bounds can be obtained for this constant, which we denote by $\lambda(K)$ (see $\S \S 2.4$ and 2.5 in [L]). For example, $\lambda(K) \leq$ $\exp (4.39(K-1))$. This bound for $M_{f}$ is not easy to obtain. The Main Lemma allows us to easily get an estimate for $M_{f}$ which has the right behaviour for $K$ close to 1 . In fact the next corollary is an immediate consequence of the uniform bound for $a$ and the fact that $\|\mu\|_{\infty} \leq(K-1)(K+1)^{-1}$.

Corollary 2.5. With notation as above, $M(f) \leq \exp (C(K)(K-1))$.

\section{THE GEOMETRY OF A-D CURVES}

In this section $\Gamma$ will be a rectifiable $\lambda$ A-D curve and the map $z: I_{0} \rightarrow \Gamma$ will denote an arclength parameterisation of $\Gamma$. We will construct a $C(\lambda)$ subdivision of $I_{0}$ that gives a 4-subdivision of $\Gamma$ and we will then use this to get the estimate (1.3) for the coefficients $\lambda_{I}$. 
Theorem 3.1. There exists a $C(\lambda)$-subdivision $\Lambda$ of $I_{0}$ such that

(i) $\operatorname{diam}(z(I)) \leq 4|z(I)|$, for any $I$ in $\Lambda$.

(ii) $\Lambda$ gives a 4-subdivision of $\Gamma$.

(iii) For any $I$ in $\Lambda,|I| \sim|z(I)|$ with a constant that depends only on $\lambda$.

We will refer to $\Lambda$ as a good subdivision of $I_{0}$.

Proof. Let $\Lambda_{0}=\left\{I_{0}\right\}$. We will define $\Lambda_{N+1}$ inductively. For $I=\left[a_{I}, b_{I}\right] \in \Lambda_{N}$ let $c_{I}$ be one of the points of $I$ whose image, $z\left(c_{I}\right)$, is at the maximal distance from the set consisting of the two points $z\left(a_{I}\right)$ and $z\left(b_{I}\right) . \Lambda_{N+1}$ is then the union over all $I \in \Lambda_{N}$ of the intervals $\left[a_{I}, c_{I}\right]$ and $\left[c_{I}, b_{I}\right]$.

We will prove (i) first. Because $z$ is a 2-parameterisation of $\Gamma$ we get that

$$
\operatorname{diam}\left(z\left(I_{0}\right)\right) \leq 2\left|z\left(I_{0}\right)\right| \leq 4\left|z\left(I_{0}\right)\right|
$$

which is (i) for $I_{0}$. Now let $I=[a, b] \in \Lambda_{N}$ for $N \geq 1$. Without loss of generality $I_{P}=[t, b]$ and so $a=c_{I_{P}}$. Let $R$ be the distance from $z(a)$ to the set $\{z(t), z(b)\} . R$ must be at least $|z(t)-z(b)| / 2$ because $I$ is connected and therefore has at least one point whose image is equidistant from $\{z(t), z(b)\}$. Clearly $R \leq|z(b)-z(a)|$. We must have

$$
z\left(I_{P}\right) \subseteq \bar{B}(z(t), R) \cup \bar{B}(z(b), R)
$$

and (i) follows easily from this using the estimates for $R$.

To show (ii) note that from (i) we get that $\left|z\left(I_{L}\right)\right|,\left|z\left(I_{R}\right)\right| \leq 4|z(I)|$. We get the opposite inequality because, as above, the connectivity of $I$ ensures that both $\left|z\left(I_{L}\right)\right|$ and $\left|z\left(I_{R}\right)\right|$ are at least $|z(I)| / 2$.

From (i), (ii), and the facts that $\Gamma$ is $\lambda$ A-D and $z$ is a 2-parameterisation of $\Gamma$, we can deduce the following sets of inequalities:

$$
\begin{aligned}
\frac{|z(I)|}{8} & \leq \frac{\left|z\left(I_{L}\right)\right|}{2} \leq \frac{H_{1}\left(z\left(I_{L}\right)\right)}{2} \leq\left|I_{L}\right|, \\
|I| & \leq 2 H_{1}(z(I)) \leq 8 \lambda|z(I)|
\end{aligned}
$$

and so we get (iii).

Finally, from (ii) and (iii) we see that $\Lambda$ is a $C(\lambda)$ subdivision of $I_{0}$.

Remarks. (a) It follows from (iii) that this subdivision has the chord-arc type property

$$
\sum_{J \subset I, J \in \Lambda_{N}}|z(J)| \leq C(\lambda)|z(I)| \quad \text { where } I \in \Lambda_{M} \text { and } N \geq M .
$$

(b) When $\Gamma$ is the unit interval then $\Lambda$ is the dyadic subdivision of the unit interval.

We need to introduce (even) more notation. Recalling that $x_{I}$ is the image of the left-hand endpoint of $I$, define $R_{I}$ and $B_{I}$ to be $8|z(I)|$ and $B\left(x_{I}, R_{I}\right)$ respectively. Note that $z(I) \subset B\left(x_{I}, \frac{5}{8} R_{I}\right)$. The next theorem is easy to see when $\Gamma$ is the unit interval (in which case $\Lambda$ is the dyadic subdivision).

Theorem 3.2. If $w \in C \backslash \Gamma$ and $\Lambda_{w}=\left\{I \in \Lambda: w \in B_{I}\right\}$ then

$$
\sum_{I \in \Lambda_{w}} \frac{1}{\left|w-z_{I}\right|^{1 / 2}} \frac{1}{R_{I}^{1 / 2}} \leq C(\lambda) \frac{1}{d(w, \Gamma)},
$$




$$
\sum_{I \notin \Lambda_{w}} \frac{R_{I}^{2}}{\left|w-z_{I}\right|^{3}} \leq C(\lambda) \frac{1}{d(w, \Gamma)},
$$

where $z_{I}$ is any element of $z(I)$. Here $d(w, \Gamma)$ is the distance from $w$ to $\Gamma$. We also have for, $I \in \Lambda$,

$$
\sum_{\substack{J \in \Lambda \\ J \subseteq I}} R_{J}^{2} \leq C(\lambda) R_{I}^{2}
$$

We will prove the theorem once we have the next lemma. Let $\mathscr{F}_{k}=\{I \in$ $\left.\Lambda: 2^{-k} \leq R_{I}<2^{-k+1}\right\}$.

Lemma 3.3. Let $x \in \Gamma$. Then the number of elements of $\mathscr{F}_{k}$ for which $z(I)$ intersects $B(x, R)$ is no more than $C(\lambda)\left[1+R 2^{k}\right]$.

Proof. Let $\Omega=\left\{I \in \mathscr{F}_{k}: z(I) \cap B(x, R) \neq \varnothing\right\}$.

Case (i) $R \leq 2^{-k}$. In this case $B\left(x, 5.2^{-k}\right)$ contains $B_{I}$, and hence $z(I)$, for all $I$ in $\Omega$. Consequently

$$
\sum_{I \in \Omega}|I| \leq 2 H_{1}\left(B\left(x, 5.2^{-k}\right)\right) \leq 10 \lambda 2^{-k} .
$$

But from Theorem 3.1 we have that $|I| \sim 2^{-k}$, hence \# $\Omega \leq C(\lambda)$.

Case (ii) $R>2^{-k}$. Then $B(x, 5 R)$ contains $z_{I}$ for all $I$ in $\Omega$ and so, as above, we get that $\# \Omega \leq C(\lambda) R 2^{k}$.

Proof of Theorem 3.2. The statement of the theorem is scale invariant, so we can assume that $d(w, \Gamma)=1$. Let $z_{0}$ be an element of $\Gamma$ with $d\left(z_{0}, w\right)=1$.

We will prove (i) first. Clearly $\Lambda_{w} \cap \mathscr{F}_{k}$ is empty for $k>0$. If $I \in \Lambda_{w} \cap \mathscr{F}_{k}$ for $k \leq 0$ then $\left|w-x_{I}\right| \leq 2.2^{-k}$ and so $\left|z_{0}-z_{I}\right|<6.2^{-k}$. Thus $z(I)$ intersects $B\left(z_{0}, 6.2^{-k}\right)$ for all $I \in \Lambda_{w} \cap \mathscr{F}_{k}$ and it follows from the previous lemma that $\#\left(\Lambda_{w} \cap \mathscr{F}_{k}\right) \leq C(\lambda)$. Then

$$
\begin{aligned}
\sum_{I \in \Lambda_{w}} \frac{1}{\left|w-z_{I}\right|^{1 / 2}} \frac{1}{R_{I}^{1 / 2}} & \leq \sum_{I \in \Lambda_{w}} \frac{1}{R_{I}^{1 / 2}} \leq \sum_{k=-\infty}^{\infty} \sum_{I \in \Lambda_{w} \cap \mathscr{F}_{k}} \frac{1}{R_{I}^{1 / 2}} \\
& \leq C(\lambda) \sum_{k \leq 0} 2^{k} \leq C(\lambda) .
\end{aligned}
$$

Now for (ii). Let $\Delta_{k}=\left\{I \in \mathscr{F}_{k}: w \notin B_{I}\right\}, A_{n}=\left\{I \in \Lambda: 2^{n-1}<\left|w-x_{I}\right| \leq\right.$ $\left.2^{n}\right\}$, for $n \geq 0$. Because $w \notin B_{I}$ we see that $\Delta_{k} \cap A_{n}=\varnothing$ for $k<-n$. Also, because $w \notin B_{I}$ we have that $\left|w-z_{I}\right| \sim\left|w-x_{I}\right|$. Thus there is a universal constant $C$ for which

$$
\begin{aligned}
\sum_{I \notin \Lambda_{w}} \frac{R_{I}^{2}}{\left|w-z_{I}\right|^{3}} & \leq C \sum_{n=0}^{\infty} \sum_{k=-n}^{\infty} \sum_{I \in \Delta_{k} \cap A_{n}} \frac{R_{I}^{2}}{\left|w-x_{I}\right|^{3}} \\
& \leq C \sum_{n=0}^{\infty} \sum_{k=-n}^{\infty} \sum_{I \in \Delta_{k} \cap A_{n}} \frac{4^{-k}}{8^{n}} .
\end{aligned}
$$

We want to use the previous lemma to estimate $\#\left(\Delta_{k} \cap A_{n}\right)$ for $k \geq-n$. If $I \in \Delta_{k} \cap A_{n}$ then

$$
\left|w-x_{I}\right| \leq 2^{n} \Rightarrow\left|z_{0}-x_{I}\right| \leq 2^{n}+1 \Rightarrow\left|z_{0}-z_{I}\right| \leq 2^{n}+1+2^{-k+1} \leq 4.2^{n}
$$


and so $\#\left(\Delta_{k} \cap A_{n}\right) \leq C(\lambda) 2^{n} 2^{k}$ for $k \geq-n$. Thus

$$
\sum_{I \notin \Lambda_{w}} \frac{R_{I}^{2}}{\left|w-z_{I}\right|^{3}} \leq C(\lambda) \sum_{n=0}^{\infty} \sum_{k=-n}^{\infty} \frac{2^{n} 2^{k} 4^{-k}}{8^{n}} \leq C(\lambda) .
$$

To show (iii), note that from (iii) in Theorem 3.1 we get that $|J| \sim R_{J}$ with a constant that depends only on $\lambda$. Thus we have to prove that $\sum_{J \in \Lambda, J \subseteq I}|J|^{2} \leq$ $C(\lambda)|I|^{2}$. This is an easy consequence of $\Lambda$ being a $C(\lambda)$ subdivision.

Recalling the definition of $\lambda_{I}$ from (1.2), the next two corollaries will give us estimates on the $\lambda_{I}$. The second one gives us the estimate (1.3), mentioned in $\S 1$.

Corollary 3.4. If $\rho$ is a quasiconformal map that fixes $\infty, \mu$ is the dilatation of $\rho, \Lambda$ is the good subdivision constructed above, and $J \in \Lambda$ then $\exists z_{J} \in z(J)$ such that

$$
\left|\lambda_{J_{L}}\right|^{2} \leq C(K)\left\{\iint_{\mathbf{C} \backslash B_{J}} \frac{|\mu|^{2} R_{J}}{\left|w-x_{J}\right|^{3}}+\iint_{B_{J}} \frac{|\mu|^{2} R_{J}^{-3 / 2}}{\left|w-z_{J}\right|^{1 / 2}}\right\} .
$$

We also have the following uniform bound on $\lambda_{J_{L}}$ : there is $A(K)$ such that

$$
\left|\lambda_{J_{L}}\right| \leq \min \left(A(K)\|\mu\|_{\infty}, C(K)\|\mu\|_{C}^{1 / 2}\right) .
$$

Proof. By compostion with translations and dilations we can assume that $x_{J}=$ $0, y_{J}=1, B_{J}=B(0,8), R_{J}=8$ and that $\rho$ fixes 0,1 and $\infty$. From the definition of $\lambda_{J_{L}}$ we see that what we need to estimate is $\rho\left(y_{J_{L}}\right)\left(y_{J_{L}}\right)^{-1}$. Because of the properties of $\Lambda$, we have $y_{J_{L}} \in B(0,5) \backslash B(0,1 / 4)$, so the first part of the corollary and the first uniform bound now follow directly from the Main Lemma.

It is easy to bound the second term in (3.1) by $\|\mu\|_{C}$. Now for the first term. Let $A_{n}=\left\{w: 2^{n} R_{J} \leq|w|<2^{n+1} R_{J}\right\}$. Then

$$
\begin{aligned}
\iint_{\mathbf{C} \backslash B_{J}} \frac{|\mu|^{2} R_{J}}{\left|w-x_{J}\right|^{3}} & \leq \sum_{n \geq 3} \frac{1}{R_{J} 4^{n}} \iint_{A_{n}} \frac{|\mu|^{2}}{d(w, \Gamma)} \\
& \leq \sum_{n \geq 3} 4^{-n}\|\mu\|_{C} 2^{n+1} \leq\|\mu\|_{C} .
\end{aligned}
$$

This gives the second uniform bound.

Corollary 3.5. If $\rho, \mu$ and $\Lambda$ are as in the previous corollary then, for $I \in \Lambda$,

$$
\sum_{\substack{J \in \Lambda \\ J \subseteq I}}\left|\lambda_{J_{L}}\right|^{2}|J| \leq C(\lambda, K)\|\mu\|_{C}|I| .
$$

Proof. Because of the properties of $\Lambda$, we get that $R_{J} \sim|J|$ and if we now use the previous corollary, we see that

$$
\sum_{\substack{J \in \Lambda \\ J \subseteq I}}\left|\lambda_{J_{L}}\right|^{2}|J| \leq C(K)\left\{\sum_{\substack{J \in \Lambda \\ J \subseteq I}} \iint_{\mathbf{C} \backslash B_{J}} \frac{|\mu|^{2} R_{J}^{2}}{\left|w-x_{J}\right|^{3}}+\sum_{\substack{J \in \Lambda \\ J \subseteq I}} \iint_{B_{J}} \frac{|\mu|^{2} R_{J}^{-1 / 2}}{\left|w-z_{J}\right|^{1 / 2}}\right\} .
$$


Let $A_{w}=\left\{J \in \Lambda: J \subseteq I\right.$ and $\left.w \in B_{J}\right\}$ and $B_{w}=\{J \in \Lambda: J \subseteq I$ and $\left.w \notin B_{J}\right\}$. There is a universal constant $\alpha$ such that if $J \subseteq I$ then $B_{J} \subseteq \alpha B_{I}$, so we can rewrite the right-hand side of (3.2) as

$$
\iint_{\mathbf{C}}|\mu|^{2}\left(\sum_{J \in B_{w}} \frac{R_{J}^{2}}{\left|w-x_{J}\right|^{3}}\right)+\iint_{\alpha B_{I}}|\mu|^{2}\left(\sum_{J \in A_{w}} \frac{R_{J}^{-1 / 2}}{\left|w-z_{J}\right|^{1 / 2}}\right) .
$$

Theorem 3.2 bounds the sum in the second term by $C(\lambda) d(w, \Gamma)^{-1}$. Thus the second term is bounded by $C(\lambda)\|\mu\|_{C} \alpha R_{I}$, which we can rewrite as $C(\lambda)\|\mu\|_{C}|I|$.

In order to estimate the first term in (3.3), we write it as

$$
\iint_{\mathbf{C} \backslash 2 \alpha B_{I}}|\mu|^{2}\left(\sum_{J \in B_{w}} \frac{R_{J}^{2}}{\left|w-x_{J}\right|^{3}}\right)+\iint_{2 \alpha B_{I}}|\mu|^{2}\left(\sum_{J \in B_{w}} \frac{R_{J}^{2}}{\left|w-x_{J}\right|^{3}}\right) .
$$

We can use Theorem 3.2 once again to show that the second term in (3.4) is bounded by $C(\lambda)\|\mu\|_{C}|I|$. From Theorem 3.2 and the fact that $\left|w-x_{J}\right| \sim$ $\left|w-x_{I}\right|$ if $w \in \mathbf{C} \backslash 2 \alpha B_{I}$, we see that the first term is bounded by

$$
\iint_{\mathbf{C} \backslash 2 \alpha B_{I}} \frac{|\mu|^{2}}{\left|w-x_{I}\right|^{3}} R_{I}^{2} \text {. }
$$

As in Corollary 3.4 , we can bound this by $C(\lambda)\|\mu\|_{C} R_{I}$ and hence by $C(\lambda)\|\mu\|_{C}|I|$.

\section{CARLESON COEFFICIENTS AND BMO FUNCTIONS}

As mentioned in $\S 1$, we want to use Corollary 3.5 to show that the $f_{N}$, as defined in $\S 1$, are in $\mathrm{BMO}(\Lambda)$. In fact we will prove the following proposition.

Proposition 4.1. If $\Gamma$ is a rectifiable $\lambda A-D$ curve, $\Lambda$ is the good subdivision constructed in the previous section, $\rho \in \Sigma_{C}$, and $f_{N}$ is as in $\S 1$ then

(i) $\exists f \in \mathrm{BMO}(\Lambda)$ such that $f_{N} \rightarrow f$ a.e., $\left\|f_{N}-f\right\|_{1} \rightarrow 0$, and

$$
\|f\|_{*} \leq C(\lambda, K)\left(\|\mu\|_{C}+\|\mu\|_{C}^{1 / 2}\right) \text {. }
$$

(ii) Each $f_{N}$ is in $\operatorname{BMO}(\Lambda)$ and $\left\|f_{N}\right\|_{*} \leq C(\lambda, K)\left(\|\mu\|_{C}+\|\mu\|_{C}^{1 / 2}\right)$.

(iii) $f_{N}$ is constant on each $I \in \Lambda_{N}$ and

$$
\begin{aligned}
& \qquad\left|f_{I}-f_{N}\right|_{I} \mid \leq C(\lambda, K)\left(\|\mu\|_{C}+\|\mu\|_{C}^{1 / 2}\right) . \\
& \text { (iv) }\left|f_{I_{0}}\right| \leq C(\lambda, K)\left(\|\mu\|_{C}+\|\mu\|_{C}^{1 / 2}\right) .
\end{aligned}
$$

The next two lemmas show how we get $\operatorname{BMO}(\Lambda)$ functions once we have estimates on the coefficients in some expansion of the function. In the first lemma the $h_{I}$ are the Haar type functions defined in $\S 1$ by

$$
h_{I}(x)=\chi_{I_{L}}(x)-\left(\left|I_{L}\right| /\left|I_{R}\right|\right) \chi_{I_{R}}(x) \text {. }
$$

Let $\Delta$ be an $\alpha$-subdivision of $I_{0}$. We say that $\left\{c_{I}\right\}_{I \in \Delta}$ are $\tau$ Carleson coefficients if

$$
\sup _{I} \frac{1}{|I|}\left(\sum_{\substack{J \subseteq I \\ J \in \Delta}}\left|c_{J}\right||J|\right) \leq \tau .
$$


Lemma 4.2. If $\left\{\left|b_{I}\right|^{2}\right\}_{I \in \Delta}$ are $\tau$ Carleson coefficients and if

$$
g_{N}(x)=\sum_{j=0}^{N-1} \sum_{I \in \Delta_{j}} b_{I} h_{I}(x) \quad \text { for } N \geq 1
$$

then

(i) $\exists g \in \mathrm{BMO}(\Delta)$ such that $g_{N} \rightarrow g$ a.e., $\left\|g_{N}-g\right\|_{2} \rightarrow 0$, and $\|g\|_{*} \leq$ $C(\alpha) \tau^{1 / 2}$.

(ii) Each $g_{N}$ is in $\mathrm{BMO}(\Delta)$ and $\left\|g_{N}\right\|_{*} \leq C(\alpha) \tau^{1 / 2}$.

(iii) $g_{N}$ is constant on each $I \in \Delta_{N}$ and $g_{I}=\left.g_{N}\right|_{I}$.

(iv) $g_{I_{0}}=0$.

Lemma 4.3. If $\Delta$ is an $\alpha$ subdivision of $I_{0}$ and $\left\{c_{I}\right\}_{I \in \Delta}$ are $\tau$ Carleson coefficients and if

$$
g_{N}(x)=\sum_{j=0}^{N-1} \sum_{I \in \Delta_{j}} c_{I} \chi_{I_{R}}
$$

then

(i) $\exists g \in \mathrm{BMO}(\Delta)$ such that $g_{N} \rightarrow g$ a.e., $\left\|g_{N}-g\right\|_{1} \rightarrow 0$, and $\|g\|_{*} \leq$ $C(\alpha) \tau$.

(ii) Each $g_{N}$ is in $\mathrm{BMO}(\Delta)$ and $\left\|g_{N}\right\|_{*} \leq C(\alpha) \tau$.

(iii) $g_{N}$ is constant on each $I \in \Delta_{N}$ and $\left|g_{I}-g_{N}\right|_{I} \mid \leq C(\alpha) \tau$.

(iv) $\left|g_{I_{0}}\right| \leq C(\alpha) \tau$.

These results basically follow from the definitions.

Proof of Proposition 4.1. From $\S 1$ we know that

$$
f_{N}(x)=\sum_{j=0}^{N-1} \sum_{I \in \Lambda_{j}} \lambda_{I_{L}} \chi_{I_{L}}(x)+\lambda_{I_{R}} \chi_{I_{R}}(x) \quad \text { a.e. }
$$

If we write

$$
\lambda_{I_{L}} \chi_{I_{L}}+\lambda_{I_{R}} \chi_{I_{R}}=\lambda_{I_{L}} h_{I}+E_{I} \chi_{I_{R}}
$$

then

$$
f_{N}(x)=\sum_{j=0}^{N-1} \sum_{I \in \Lambda_{j}} \lambda_{I_{L}} h_{I}(x)+\sum_{j=0}^{N-1} \sum_{I \in \Lambda_{j}} E_{I} \chi_{I_{R}}(x) \quad \text { a.e. }
$$

Corollary 3.5 tells us that $\left\{\left|\lambda_{I_{L}}\right|^{2}\right\}$ are $C(\lambda, K)\|\mu\|_{C}$ Carleson coefficients and if we knew that the $E_{I}$ were also Carleson coefficients then the proposition would follow immediately from the previous two lemmas.

It follows easily from the definitions that

$$
\exp \left(\lambda_{I_{R}}\right)-1+z_{I_{R}}^{-1} z_{I_{L}}\left(\exp \left(\lambda_{I_{L}}\right)-1\right)=0
$$

where $z_{J}=z(b)-z(a)$ for $J=[a, b]$. We can rewrite this as

$$
\lambda_{I_{R}}+z_{I_{R}}^{-1} z_{I_{L}} \lambda_{I_{L}}=\left(1+\lambda_{I_{R}}-\exp \left(\lambda_{I_{R}}\right)\right)+z_{I_{R}}^{-1} z_{I_{L}}\left(1+\lambda_{I_{L}}-\exp \left(\lambda_{I_{L}}\right)\right) .
$$

If we denote the right-hand side of this equality by $e_{I}$, then

$$
\left|e_{I}\right| \leq C(\lambda, K)\left(\left|\lambda_{I_{L}}\right|^{2}+\left|\lambda_{I_{R}}\right|^{2}\right) \text {. }
$$


Going back to (4.2) we find that

$$
E_{I}=\lambda_{I_{L}} \frac{\left|I_{L}\right|}{\left|I_{R}\right|}\left(1-\frac{\left|I_{R}\right|}{\left|I_{L}\right|} z_{I_{R}}^{-1} z_{I_{L}}\right)+e_{I}
$$

For $J \in \Lambda$ we make the following definitions:

$$
Q(J)=\frac{z_{J}}{|J|}, \quad D(J)=1-\frac{Q\left(J_{L}\right)}{Q\left(J_{R}\right)}, \quad \text { and } \quad d_{J}=\lambda_{J_{L}} \frac{\left|J_{L}\right|}{\left|J_{R}\right|} D(J) .
$$

Then $E_{I}=e_{I}+d_{I}$. It follows from (4.4) and Corollary 3.5 that the $e_{I}$ are $C(\lambda, K)\|\mu\|_{C}$ Carleson coefficients. Next, we will show that the $|D(I)|^{2}$ are Carleson coefficients and we will deduce that the $d_{I}$ are also Carleson coefficients. This will finish the proof of the proposition.

It follows from the properties of $\Lambda$ that $|Q(J)| \geq 1 / C(\lambda)$. Therefore

$$
|D(J)| \leq C(\lambda)\left|Q\left(J_{L}\right)-Q\left(J_{R}\right)\right| \text {. }
$$

Let $I \in \Lambda$ and define $h=\left(z^{\prime}-Q(I)\right) \chi_{I}$. Then $\int_{I} h=0$, so

$$
h=\sum_{\substack{J \in \Lambda \\ J \subseteq I}}\left(h, h_{J}\right) \frac{h_{J}}{\left(h_{J}, h_{J}\right)} .
$$

From the definitions of $h, h_{J}$ we get that

$$
\left(h, h_{J}\right)=\left|J_{L}\right|\left(Q\left(J_{L}\right)-Q\left(J_{R}\right)\right) \quad \text { and } \quad \frac{|J|}{C(\lambda)} \leq\left(h_{J}, h_{J}\right) \leq C(\lambda)|J| .
$$

Therefore

$$
C(\lambda)|I| \geq\|h\|_{2}^{2} \geq \frac{1}{C(\lambda)} \sum_{\substack{J \in \Lambda \\ J \subseteq I}}\left|J_{L}\right|\left|Q\left(J_{L}\right)-Q\left(J_{R}\right)\right|^{2} \geq \frac{1}{C(\lambda)} \sum_{\substack{J \in \Lambda \\ J \subseteq I}}|J||D(J)|^{2} .
$$

In other words, the $|D(J)|^{2}$ are $C(\lambda)$ Carleson coefficients. Then, because

$$
\begin{aligned}
\sum_{\substack{J \in \Lambda \\
J \subseteq I}}\left|d_{J}\right||J| & \leq C(\lambda) \sum_{\substack{J \in \Lambda \\
J \subseteq I}}\left|\lambda_{J_{L}}\right||D(J)||J| \\
& \leq C(\lambda)\left(\sum_{\substack{J \in \Lambda \\
J \subseteq I}}\left|\lambda_{J_{L}}\right|^{2}|J|\right)^{1 / 2}\left(\sum_{\substack{J \in \Lambda \\
J \subseteq I}}|D(J)|^{2}|J|\right)^{1 / 2}
\end{aligned}
$$

we see that the $d_{I}$ are $C(\lambda, K)\|\mu\|_{C}^{1 / 2}$ Carleson coefficients.

\section{BMO OF A-D CURVES}

BMO of a locally rectifiable curve was defined in the introduction. In the next section we will need to be able to identify exponentials of functions with small BMO norm. Theorem 5.3 will allow us to do this. We will need the following easily checked fact: If $\Gamma$ is a rectifiable $\lambda$ A-D curve, $z: I_{0} \rightarrow \Gamma$ is an arclength parameterisation of $\Gamma$, and $f \in \operatorname{BMO}(\Gamma)$ then $f \circ z \in \operatorname{BMO}\left(I_{0}\right)$ and $\|f \circ z\|_{*} \leq c(\lambda)\|f\|_{*}$. 
Theorem 5.1 (John-Nirenberg). Let $\Gamma$ be a $\lambda$ A-D curve. If $f \in \mathrm{BMO}(\Gamma)$ and $B$ is a ball centered on $\Gamma$ then for any $t \geq 0$

$$
\frac{H_{1}\left\{x \in B \cap \Gamma:\left|f(x)-f_{B}\right|>t\right\}}{H_{1}(B \cap \Gamma)} \leq C(\lambda) \exp \left(\frac{-b(\lambda) t}{\|f\|_{*}}\right) .
$$

Proof. Proposition 1.4 gives us that $\Gamma \cap B \subseteq \bigcup_{n=1}^{N(\lambda)} \gamma_{n}$, where each $\gamma_{n}$ is a $\lambda$ A-D curve contained in $\Gamma \cap 2 B$ and $H_{1}\left(\gamma_{n}\right) \sim H_{1}(\Gamma \cap B)$ with a constant that depends only on $\lambda$. Let $f_{n}$ be $f$ restricted to $\gamma_{n}, z_{n}: I_{n} \rightarrow \gamma_{n}$ be the arclength parameterisation of $\gamma_{n}$ and $g_{n}$ be $f_{n} \circ z_{n}$. Let $a_{n}$ be the average value of $g_{n}$ over $I_{n}$. Each $g_{n}$ is in BMO and because $f \in$ BMO we can uniformly bound $\left|a_{n}-f_{B}\right|$. Now use the usual John-Nirenberg Theorem for each $g_{n}$ and put the estimates together to get the result.

The same approach can be used to prove the next theorem.

Theorem 5.2 (Stromberg). Let $\Gamma$ be a $\lambda$ A-D curve. $\exists \alpha_{0}(\lambda)$ such that if $f$ is a measurable function on $\Gamma$ and $\exists \alpha<\alpha_{0}(\lambda), \beta>0$ such that for any ball $B$ centered on $\Gamma$ there exists a constant $a_{B}$ for which

$$
H_{1}\left\{x \in \Gamma \cap B:\left|f(x)-a_{B}\right|>\beta\right\} \leq \alpha H_{1}(\Gamma \cap B)
$$

tinen $f \in \mathrm{BMO}(\Gamma)$ and $\|f\|_{*} \leq C(\lambda) \beta$.

Theorem 5.3. Let $\Gamma$ be a $\lambda$ A-D curve. $\exists \delta_{0}(\lambda)$ with the following property: Let $F$ be a measurable function on $\Gamma$ which is not identically zero on any ball. Suppose $\exists \delta \leq \delta_{0}$ such that for any ball $B$ centered on $\Gamma$ there is a constant $c_{B}$ for which

$$
\frac{\int_{\Gamma \cap B}\left|F-c_{B}\right|}{H_{1}(\Gamma \cap B)} \leq \delta \frac{\int_{\Gamma \cap B}|F|}{H_{1}(\Gamma \cap B)} .
$$

Then $F=e^{f}$ a.e., where $f \in \operatorname{BMO}(\Gamma)$ and $\|f\|_{*} \leq C(\lambda) \delta$.

Semmes proved this theorem on $\mathbf{R}$ (see [S1]) and the proof on $\Gamma$ is exactly the same provided we know the following lemma.

Lemma 5.4. $\exists \delta_{0}(\lambda)$ such that if $|u| \equiv 1$ and $\|u\|_{*} \leq \delta_{0}(\lambda)$, then there exists $f \in \mathrm{BMO}(\Gamma)$ such that $u=e^{f}$ a.e. and $\|f\|_{*} \leq C(\lambda)\|u\|_{*}$.

We will prove the lemma initially for the case where $\Gamma$ is compact. There is no loss of generality if we assume that $\Gamma$ has diameter $1 / 2$. In the following we will assume that $u$ is as in the statement of the lemma and that $\delta_{0}(\lambda)$ is always small enough for the various steps to work.

For any $x \in \Gamma$ we define $u_{N}(x)$ for $N \geq 0$ to be the average value of $u$ over $B_{N}(x)$. The proof of the Lebesgue differentiation theorem on $\mathbf{R}$ (see [St]) works equally well on $\Gamma$, so we get that $u_{N}(x)$ converges to $u(x)$ a.e. on $\Gamma$. Note that $u_{0}(x)$ is the same for all $x$. We will call this value $u_{0}$. The following are easy to obtain from the facts that $u$ is in BMO and that $\Gamma$ is A-D:

(i) $\left|u_{N}(x)-u_{N+1}(x)\right| \leq 2 \lambda\|u\|_{*}$.

(ii) $1-\|u\|_{*} \leq\left\|u_{N}\right\|_{\infty} \leq 1$.

Define $\arg (z), z \in C \backslash\{0\}$, so that the discontinuity is along the negative real axis. Then define $f_{0}(x)=i \arg \left(u_{0}\right)$ and $f_{N+1}(x)=f_{N}(x)+i \arg \left(u_{N+1}(x) / u_{N}(x)\right)$. The next two lemmas show how the convergence of the $u_{N}$ is reflected in the $f_{N}$. 
Lemma 5.5. Fix an $n \geq 0$. If $\left|u_{m}(x)-u_{n}(x)\right| \leq \delta \leq \pi / 8$ for $n \leq m$ then $\left|f_{m}(x)-f_{n}(x)\right| \leq 6 \delta$ for $n \leq m$.

Proof. It is easily seen that

$$
\left|e^{f_{n}(x)}-e^{f_{m}(x)}\right| \leq 3\left|u_{m}(x)-u_{n}(x)\right| \leq 3 \delta .
$$

Thus $\left|f_{m}(x)-f_{n}(x)-2 \pi N(m)\right| \leq 6 \delta$ for some $N(m) \in Z$. However, from the definition of the $f_{m}$ and the bound on $\left|u_{m}(x)-u_{n}(x)\right|$ we get that $\mid f_{m}(x)-$ $f_{m+1}(x) \mid \leq 6 \delta$ for any $m$, and because $\delta \leq \pi / 8$ we see that $N(m)=N(n)=0$ for $n \leq m \leq N_{0}$.

Lemma 5.6. If $y \in B_{N}(x)$ then $\left|f_{m}(y)-f_{m}(x)\right| \leq C(\lambda)\|u\|_{*}$ for $0 \leq m \leq N$.

Proof. If $y \in B_{N}(x)$ then $B_{m}(y) \subseteq B_{m-1}(x)$, therefore $\left|u_{m}(y)-u_{m-1}(x)\right| \leq$ $C(\lambda)\|u\|_{*}$. Thus $\left|u_{m}(y)-u_{m}(x)\right| \leq C(\lambda)\|u\|_{*}$ for $1 \leq m \leq N$. Now the same proof as the previous lemma works, provided $\|u\|_{*}$ is small enough, because $f_{0}(x)=f_{0}(y)$.

Proof of Lemma 5.4 (compact case). As a direct consequence of Lemma 5.5 we get that the $f_{N}$ converge a.e. to a measurable function $f$ and $e^{f}=u$ a.e. We are going to use Stromberg's theorem to show that $f$ is in $\operatorname{BMO}(\Gamma)$.

Let $x \in \Gamma$ and $R>0$. Choose $N$ so that $2^{-N-1} \leq R \leq 2^{-N}$. Let $B=B\left(x, 2^{-N}\right)$. We will apply a stopping time process to $\left|\bar{u}-u_{B}\right|$. Let $\alpha_{M}(y)$ be the average of $\left|u-u_{B}\right|$ over $B\left(y, 2^{-M}\right)$. Then $\alpha_{N}(y) \leq C(\lambda)\|u\|_{*}$.

$$
\begin{aligned}
& \Omega_{1}=\left\{y \in B \cap \Gamma: \alpha_{M}(y) \leq A(\lambda)\|u\|_{*}, \forall M \geq N\right\}, \\
& \Omega_{2}=\left\{y \in B \cap \Gamma: \exists M \geq N \text { such that } \alpha_{M}(y)>A(\lambda)\|u\|_{*}\right\} .
\end{aligned}
$$

$A(\lambda)$ is some large constant that we will choose later.

For all $y \in \Omega_{1}$ we have that $\left|u_{M}(y)-u_{B}\right| \leq C(\lambda)\|u\|_{*}$ for $M \geq N$, so $\left|u_{M}(y)-u_{N}(y)\right| \leq C(\lambda)\|u\|_{*}$ for $M \geq N$. Now the two preceding lemmas imply that $\left|f_{M}(y)-f_{N}(x)\right| \leq C(\lambda)\|u\|_{*}$ for $M \geq N$ and so $\left|f(y)-f_{N}(x)\right| \leq C(\lambda)\|u\|_{*}$ for a.e. $y \in \Omega_{1}$. If we can show that $\Omega_{2}$ has small measure then, by Stromberg, we are done. $\Omega_{2}$ is covered by a family of balls and for each ball, $\mathscr{B}$, we have

$$
\|u\|_{*} H_{1}(\mathscr{B} \cap \Gamma) \leq \frac{1}{A(\lambda)} \int_{\mathscr{B} \cap \Gamma}\left|u-u_{B}\right| .
$$

By the Besicovitch Covering Lemma, there is a subfamily $\left\{B_{n}\right\}_{n=1}^{\infty}$ that covers $\Omega_{2}$ and for which $\sum_{n=1}^{\infty} \chi_{B_{n}} \leq C$. Thus by (5.5),

$$
\|u\|_{*} H_{1}\left(\Omega_{2}\right) \leq \frac{C}{A(\lambda)} \int_{2 B \cap \Gamma}\left|u-u_{B}\right|
$$

because each ball is contained in $2 B$. Combining this with the definition of BMO and $\Gamma$ being A-D we get

$$
H_{1}\left(\Omega_{2}\right) \leq(C(\lambda) / A(\lambda)) H_{1}(B \cap \Gamma) .
$$

By choosing $A(\lambda)$ large enough we can apply Stromberg to get that $\|f\|_{*} \leq$ $C(\lambda)\|u\|_{*}$.

This finishes the proof for the compact case.

Now let $\Gamma$ be an arbitrary $\lambda$ A-D curve and $x \in \Gamma$. Then $\Gamma=\bigcup_{N=1}^{\infty} C_{N}$, where each $C_{N}$ is the component of $\Gamma \cap B(x, N)$ that contains $x$. Thus each $C_{N}$ is a compact A-D curve and $C_{N} \subseteq C_{N+1}$. Because we have the result for 
the compact case, for each $N$ we obtain a function $g_{N} \in \operatorname{BMO}\left(C_{N}\right)$ such that $e^{g_{N}}=u$ and $\left\|g_{N}\right\|_{*} \leq C(\lambda)\|u\|_{*}$. Let $h=\left(g_{2}-g_{1}\right) / 2 \pi$. Then $h$ is integer valued on $C_{1}$ and $\|h\|_{*} \leq C(\lambda)\|u\|_{*}$. From this we can deduce that $h$ is constant, provided $\|u\|_{*}$ is small enough.

Now let $f_{1}=g_{1}$ and $f_{2}=g_{2}-2 \pi h$. Then $\left\|f_{2}\right\|_{*}=\left\|g_{2}\right\|_{*}, f_{2}=f_{1}$ on $C_{1}$, and $e^{f_{2}}=u$ on $C_{2}$. Proceed in this way to obtain $\left\{f_{N}\right\}$ such that for all $N \geq 0\left\|f_{N}\right\|_{*}=\left\|g_{N}\right\|_{*}, f_{N+1}=f_{N}$ on $C_{N}$, and $e^{f_{N}}=u$ on $C_{N}$. Define $f(z)$ to be $f_{N}(z)$ for $z \in C_{N}$. Then $f$ is well defined, $e^{f}=u$ on $\Gamma$, and $\|f\|_{*} \leq C(\lambda)\|u\|_{*}$.

\section{THE THEOREMS}

Before proceeding with the proofs of the theorems we will need the following corollary of the Main Lemma.

Lemma 6.1. Let $\rho$ be a $K$-quasiconformal mapping with dilatation $\mu$ that fixes $\infty$. If $z, w, x, y \in \mathbf{C}$ satisfy

$$
\frac{1}{\eta} \leq \frac{|x-y|}{|z-w|} \leq \eta \quad \text { and } \quad \frac{|z-x|}{|z-w|} \leq \eta
$$

for some $\eta>1$, then there exists $A(K, \eta)$ such that

$$
\left(\frac{z-w}{\rho(z)-\rho(w)}\right)\left(\frac{\rho(x)-\rho(y)}{x-y}\right)=e^{\sigma}
$$

and

$$
|\sigma| \leq \min \left(A(K, \eta)\|\mu\|_{\infty}, C(K, \eta)\|\mu\|_{C}^{1 / 2}\right) .
$$

Proof. By rescaling we may assume that $z=0, w=1$, and that $\rho$ fixes 0,1 . There is no loss of generality if we assume that $|y| \leq|x|$. Then $1 / 2 \eta \leq|x| \leq \eta$. The proofs of the Main Lemma and of Corollary 3.4 give that

$$
\rho(x) / x=e^{b}
$$

with $|b| \leq \min \left(A(K, \eta), C(K, \eta)\|\mu\|_{C}^{1 / 2}\right)$. Define

$$
\psi(z)=\frac{\rho(x)-\rho(x(1-z))}{\rho(x)} .
$$

Then $\psi$ fixes 0,1 , and $\infty$. If we take $z_{0}=(x-y) x^{-1}$ we have $\eta^{-2} \leq\left|z_{0}\right| \leq 2$ and so we get

$$
\frac{\rho(x)-\rho(y)}{x-y} \frac{x}{\rho(x)}=\frac{\psi\left(z_{0}\right)}{z_{0}}=e^{c}
$$

with $c$ having the same type of bound as $b$. If we combine this with (6.1), we obtain the lemma.

Finally, we are ready to prove the main theorems.

Theorem 6.2. If $\Gamma_{0}$ is a $\lambda$ Ahlfors-David curve (not necessarily compact) and $\rho$ is a $K$-quasiconformal map that fixes $\infty$ and whose dilatation, $\mu$, satisfies $\|\mu\|_{C}<\infty$ relative to $\Gamma_{0}$ then

(i) $\rho$ is differentiable a.e. on $\Gamma_{0}$.

(ii) $\left|\rho^{\prime}\right|>0$ a.e., $\psi=\log \left|\rho^{\prime}\right| \in \operatorname{BMO}\left(\Gamma_{0}\right)$, and

$$
\|\psi\|_{*} \leq C(\lambda, K)\left(\|\mu\|_{C}+\|\mu\|_{C}^{1 / 2}\right) .
$$


(iii) If $\Gamma_{0}=\rho\left(\Gamma_{0}\right)=\mathbf{R}$ or $\Gamma_{0}=\rho\left(\Gamma_{0}\right)=\mathbf{T}$ then $\rho$ is locally absolutely continuous, $\left|\rho^{\prime}\right| \in A_{\infty}$, and the $A_{\infty}$ norm is bounded by $A e^{C(K)\left(\|\mu\|_{C}+\|\mu\|_{C}^{1 / 2}\right)}$.

Proof. Let $B$ be any ball centered on $\Gamma_{0}$. There is a compact $\lambda$ Ahlfors-David curve $\Gamma$ contained in $\Gamma_{0}$, for which $\Gamma_{0} \cap B \subseteq \Gamma$. Let $z: I_{0} \rightarrow \Gamma$ be an arclength parameterisation and $\Lambda$ the good subdivision constructed previously. We will show that $\rho \circ z$ is differentiable a.e. on $I_{0}$. Then Proposition 1.2 gives us that $\rho$ is differentiable a.e. on $\Gamma$ and hence on $\Gamma_{0}$.

If we can show that for a.e. $x \in I_{0}$

$$
\rho(z(y))-\rho(z(x))=O(|x-y|) \text { as } y \rightarrow x
$$

then $\rho \circ z$ is differentiable a.e. (see Theorem 3, Chapter VIII of [St]) and we are done. From the definition of the $f_{N}$ we get that

$$
\frac{\rho(z(b))-\rho(z(a))}{z(b)-z(a)}=Q_{\rho}(I)=Q_{\rho}\left(I_{0}\right) e^{f_{N} \mid I}
$$

if $I=[a, b] \in \Lambda_{N}$. Using (iii) in Proposition 4.1 we can rewrite this as

$$
\rho(z(b))-\rho(z(a))=O\left(e^{f_{I}}|z(b)-z(a)|\right) .
$$

If $A_{M}=\left\{x:\left|f_{I}\right| \leq M \quad \forall I \in \Lambda\right.$ which contain $\left.x\right\}$ then a simple stopping time argument shows that $\left|I_{0} \backslash A_{M}\right| \leq(\alpha / M)\|f\|_{1}$ and so $A=\bigcup_{M=1}^{\infty} A_{M}$ has full measure in $I_{0}$. If $x \in A$ and $I=[a, b] \in \Lambda$ contains $x$ then

$$
\rho(z(b))-\rho(z(a))=O(|z(b)-z(a)|) .
$$

Let $y \in I_{0}$. If $z(y)=z(x)$ then we certainly have

$$
\rho(z(y))-\rho(z(x))=O(|z(y)-z(x)|)
$$

so we will assume that $z(y) \neq z(x)$.

Because $\left|z\left(I_{0}\right)\right| \sim \operatorname{diam}(\Gamma),|J| \sim|z(J)|$ for all $J \in \Lambda$ and $\Lambda$ is a $C(\lambda)$ subdivision, there must exist $I=[a, b] \in \Lambda$ which contains $x$ and for which $|z(I)| \sim|z(y)-z(x)|$ with a constant that depends only on $\lambda$. Because $\operatorname{diam}(z(I)) \leq 4|z(I)|$ we also get that $z(a), z(b)$ are a bounded distance from $z(x)$. Thus the points $z(a), z(b), z(y), z(x)$ satisfy the conditions of Lemma 6.1. Consequently

$$
\frac{1}{A(\lambda, K)} \leq\left|\frac{\rho(z(x))-\rho(z(y))}{z(y)-z(x)}\right|\left|\frac{z(b)-z(a)}{\rho(z(b))-\rho(z(a))}\right| \leq A(\lambda, K) .
$$

If we combine this with $(6.3)$ we get

$$
\rho(z(x))-\rho(z(y))=O(|z(y)-z(x)|)=O(|x-y|),
$$

which concludes the proof of (i).

Now for (ii). Let $B$ be a ball centered on $\Gamma_{0}$. We can write

$$
\Gamma_{0} \cap B \subseteq \bigcup_{n=1}^{N(\lambda)} \gamma_{n}
$$

where the $\gamma_{n}$ are as in Proposition 1.4.

Let $\gamma$ be one of the $\gamma_{n}$ and let $z: I_{0} \rightarrow \gamma$ be an arclength parameterisation. Then by Proposition 4.1, the fact that $\rho^{\prime}$ exists a.e. and the discussion in $\S 1$ we have

$$
\rho^{\prime} \circ z=Q_{\rho}\left(I_{0}\right) e^{f} \quad \text { a.e. }
$$


where $f$ is as in Proposition 4.1. Clearly $\left|\rho^{\prime}\right|>0$ a.e. Then

$$
\psi \circ z=\log \left|Q_{\rho}\left(I_{0}\right)\right|+\operatorname{Re} f .
$$

Let $\alpha=\log \left|Q_{\rho}\left(I_{0}\right)\right|$. It follows from Proposition 4.1 and the fact that $z$ is a 2-parameterisation that

$$
\int_{\gamma}|\psi-\alpha| \leq 2 \int_{I_{0}}|\psi \circ z-\alpha| \leq 2 \int_{I_{0}}|f| \leq C(\lambda, K) H_{1}\left(\Gamma_{0} \cap B\right)\left(\|\mu\|_{C}+\|\mu\|_{C}^{1 / 2}\right) .
$$

If we use the subscript $n$ in the obvious way, we get that

$$
\begin{aligned}
\int_{\Gamma_{0} \cap B}\left|\psi-\alpha_{1}\right| & \leq \sum_{n=1}^{N(\lambda)} \int_{\gamma_{n}}\left|\psi-\alpha_{n}\right|+\sum_{n=1}^{N(\lambda)}\left|\alpha_{n}-\alpha_{1}\right| H_{1}\left(\Gamma_{0} \cap B\right) \\
& \leq C(\lambda, K) H_{1}\left(\Gamma_{0} \cap B\right)\left(\left(\|\mu\|_{C}+\|\mu\|_{C}^{1 / 2}\right)+\sup _{n}\left|\alpha_{n}-\alpha_{1}\right|\right) .
\end{aligned}
$$

We will bound $\left|\alpha_{n}-\alpha_{1}\right|$ using Lemma 6.1. Taking $I_{0}^{n}=\left[a_{n}, b_{n}\right]$ to be the interval we use to parameterise $\gamma_{n}$ and $z_{n}$ to be an arclength parameterisation of $\gamma_{n}$, we have

$$
\alpha_{n}-\alpha_{1}=\log \left|\frac{Q_{\rho}\left(I_{0}^{n}\right)}{Q_{\rho}\left(I_{0}^{1}\right)}\right|=\log \left|\frac{\rho\left(z_{n}\left(b_{n}\right)\right)-\rho\left(z_{n}\left(a_{n}\right)\right)}{z_{n}\left(b_{n}\right)-z_{n}\left(a_{n}\right)}\right|\left|\frac{z_{1}\left(b_{1}\right)-z_{1}\left(a_{1}\right)}{\rho\left(z_{1}\left(b_{1}\right)\right)-\rho\left(z_{1}\left(a_{1}\right)\right)}\right| .
$$

The points $z_{n}\left(b_{n}\right), z_{n}\left(a_{n}\right), z_{1}\left(b_{1}\right), z_{1}\left(a_{1}\right)$ satisfy the conditions of the lemma with $\eta$ depending only on $\lambda$, so $\left|\alpha_{n}-\alpha_{1}\right| \leq C(\lambda, K)\|\mu\|_{C}^{1 / 2}$. Then from (6.5), we get

$$
\|\psi\|_{*} \leq C(\lambda, K)\left(\|\mu\|_{C}+\|\mu\|_{C}^{1 / 2}\right) .
$$

This finishes (ii).

We will prove (iii) for $\Gamma_{0}=\mathbf{R}$. The other case is similar. Because $\rho$ maps $\mathbf{R}$ onto itself there is no loss of generality if we assume that $\rho$ is increasing, hence $\rho^{\prime}>0$ a.e. Let $I_{0}$ be an interval contained in $\mathbf{R}$. We know that we can write

$$
\rho^{\prime}=Q_{\rho}\left(I_{0}\right) e^{f} \quad \text { a.e. }
$$

where $f$ is as in Proposition 4.1 with $\Lambda$ being the dyadic subdivision of $I_{0}$. Clearly $f$ is real valued. Because $\rho$ is an increasing homeomorphism of the real line we get that

$$
\int_{I_{0}} \rho^{\prime} /\left|I_{0}\right| \leq Q_{\rho}\left(I_{0}\right)
$$

From this we immediately deduce that

$$
\frac{\int_{I_{0}} \rho^{\prime}}{\left|I_{0}\right|}\left(\frac{\int_{I_{0}}\left(\rho^{\prime}\right)^{-1 / p-1}}{\left|I_{0}\right|}\right)^{p-1} \leq\left(\int_{I_{0}} \frac{e^{-f / p-1}}{\left|I_{0}\right|}\right)^{p-1}
$$

Using Proposition 1.5, the estimate for $f_{I_{0}}$ in Proposition 4.1, and choosing $p$ large enough we see that the right-hand side is bounded by $A e^{C(K)\left(\|\mu\|_{C}+\|\mu\|_{C}^{1 / 2}\right)}$. It remains to show that $\rho$ is absolutely continuous. From (6.2) we know that if $I=[a, b]$ is a dyadic interval then

$$
\rho(b)-\rho(a)=O\left(e^{f_{I}}|\bar{I}|\right) .
$$


It follows from Jensen's inequality that $|I| e^{f_{I}} \leq \int_{I} e^{f}$, hence

$$
|\rho(b)-\rho(a)| \leq O\left(\int_{I} e^{f}\right) .
$$

Combining this with the fact that $e^{f}$ is in $L^{1}$, by (6.6), we see that $\rho$ is absolutely continuous.

Theorem 6.3. Let $\Gamma_{0}, \rho, \mu$, and $K$ be as in the Theorem 6.2. There exists $\varepsilon_{0}=\varepsilon_{0}(\lambda, K)$ such that if $\|\mu\|_{C} \leq \varepsilon_{0}$ then

(i) $\rho$ is locally absolutely continuous on $\Gamma_{0}$.

(ii) $\rho$ is differentiable a.e. on $\Gamma_{0}$.

(iii) $\rho^{\prime}=e^{\phi}$ where $\phi \in \operatorname{BMO}\left(\Gamma_{0}\right)$ and $\|\phi\|_{*} \leq C(\lambda, K)\|\mu\|_{C}^{1 / 2}$.

Proof. Let $\Gamma$ be any rectifiable curve contained in $\Gamma_{0}$. Then $\Gamma$ is a rectifiable $\lambda$ A-D curve. By Proposition 1.2 we only need to check that $\rho \circ z$ is absolutely continuous, where $z$ is an arclength parameterisation of $\Gamma$. We are now in a position to use Proposition 4.1. If $I=[a, b] \in \Lambda_{N}$ then

$$
\begin{aligned}
|\rho(z(b))-\rho(z(a))| & =\left|Q_{\rho}(I)\right||z(I)|=|z(I)|\left|Q_{\rho}\left(I_{0}\right)\right|\left|\exp \left(\left.f_{N}\right|_{I}\right)\right| \\
& \leq C\left(\lambda, K,\|\mu\|_{C}\right)|I|\left|Q_{\rho}\left(I_{0}\right)\right|\left|e^{f_{I}}\right| .
\end{aligned}
$$

By Proposition 4.1, if $\|\mu\|_{C}$ is small enough then $\|f\|_{*}$ is small. Therefore, by Proposition 1.5,

Thus

$$
e^{f} \in L^{1} \quad \text { and } \quad|I|\left|e^{f_{I}}\right| \leq C(\lambda) \int_{I}\left|e^{f}\right|
$$

$$
\left|Q_{\rho}(I)\right||z(I)| \leq C(\lambda, K)\left|Q_{\rho}\left(I_{0}\right)\right| \int_{I}\left|e^{f}\right| .
$$

Because $e^{f} \in L^{1}$ the absolute continuity of $\rho \circ z$ follows.

(ii) is true because $\rho$ is locally absolutely continuous or by the previous theorem.

We will use Theorem 5.3 to prove (iii). Let $B$ be a ball centered on $\Gamma_{0}$ and let $\gamma_{n}, z_{n}, I_{0}^{n}, \gamma, I_{0}$, and $f$ be as in the proof of (ii) in the last theorem. Let $\beta=Q_{\rho}\left(I_{0}\right)$. Then

$$
\int_{\gamma}\left|\rho^{\prime}-\beta\right| \leq 2|\beta| C(\lambda, K) \int_{I_{0}}\left|e^{f}-1\right| \leq|\beta| C(\lambda, K) H_{1}\left(\Gamma_{0} \cap B\right)\|\mu\|_{C}^{1 / 2} .
$$

The second inequality following from Proposition 1.5 and Proposition 4.1. If $\beta_{n}$ is the $\beta$ for $\gamma_{n}$, it follows from what we did in the last theorem that $\left|\beta_{n} \beta_{1}^{-1}-1\right|$ is bounded by $C(\lambda, K)\|\mu\|_{C}^{1 / 2}$, if $\|\mu\|_{C}$ is small enough. Combine this with (6.7) to get

$$
\int_{\Gamma_{0} \cap B}\left|\rho^{\prime}-\beta_{1}\right| \leq C(\lambda, K) H_{1}\left(\Gamma_{0} \cap B\right)\|\mu\|_{C}^{1 / 2}\left|\beta_{1}\right| .
$$

If $\|\mu\|_{C}$ is small enough, then it follows from (6.8) that

$$
\int_{\Gamma_{0} \cap B}\left|\rho^{\prime}-\beta_{1}\right| \leq C(\lambda, K)\|\mu\|_{C}^{1 / 2} \int_{\Gamma_{0} \cap B}\left|\rho^{\prime}\right| .
$$

This is exactly one of the conditions that $\rho^{\prime}$ needs to satisfy if we want to apply Theorem 5.3. The other condition, that $\rho^{\prime}$ is not identically zero in any ball, is shown in Theorem 6.2, and thus (iii) follows if $\|\mu\|_{C}$ is small enough. 
Corollary 6.4. $\rho\left(\Gamma_{0}\right)$ is a $C(\lambda, K) A-D$ curve when $\rho$ satisfies the conditions of Theorem 6.3.

Proof. From the last theorem and using the quasiconformal distortion theorems we get the estimate

$$
\begin{aligned}
H_{1}\left(\rho\left(\Gamma_{0} \cap B\right)\right) & \leq \int_{\Gamma_{0} \cap B}\left|\rho^{\prime}\right| \leq C(\lambda, K) H_{1}\left(\Gamma_{0} \cap B\right)\left|\beta_{1}\right| \\
& \leq C(\lambda, K) H_{1}\left(\Gamma_{0} \cap B\right) \frac{\operatorname{diam} \rho\left(\Gamma_{0} \cap B\right)}{\operatorname{diam} B} .
\end{aligned}
$$

It is not too difficult to combine this with the quasiconformal distortion theorems to prove the result.

Corollary 6.5. If $\rho$ satisfies the conditions of Theorem 6.3 and $\Gamma_{0}$ is $M$ chordarc then $\rho\left(\Gamma_{0}\right)$ is $C(M, K)$ chord-arc.

Proof. $\rho\left(\Gamma_{0}\right)$ is a quasicircle and a $C(M, K)$ A-D curve. Any curve that is a quasicircle and an A-D curve is chord-arc.

The next theorem says that, up to a dilation, $\rho$ is bi-Lipschitz most of the time on any given ball.

Theorem 6.6. Let $\rho, \mu, \Gamma_{0}$ and $K$ be as in Theorem 6.2. Then given $\varepsilon>0$ there exists $M=M\left(\lambda, K, \varepsilon,\|\mu\|_{C}\right)$ such that, if $B$ is any ball centered on $\Gamma_{0}$ there is a measurable set $W \subseteq B$ for which

$$
\frac{H_{1}\left(\left(\Gamma_{0} \cap B\right) \backslash W\right)}{H_{1}\left(\Gamma_{0} \cap B\right)} \leq \varepsilon \text { and }
$$

$$
\left.\frac{\operatorname{diam}\left(\Gamma_{0} \cap B\right)}{\operatorname{diam}\left(\rho\left(\Gamma_{0} \cap B\right)\right)} \rho\right|_{W} \text { is } M \text { bi-Lipschitz. }
$$

Proof. We can assume that $\operatorname{diam}\left(\Gamma_{0} \cap B\right)=\operatorname{diam}\left(\rho\left(\Gamma_{0} \cap B\right)\right)=1$. Once again we take $\gamma, z, I_{0}$, and the $\gamma_{n}$ to be as in the proof of (ii) of Theorem 6.2. Because $\operatorname{diam}(\gamma) \sim 1$ we get that $\operatorname{diam}(\rho(\gamma)) \sim 1$, with a constant that depends only on $K$, so $Q_{\rho}\left(I_{0}\right) \sim 1$ with a constant that depends only on $K$. From Proposition 4.1 we know that we can write

$$
Q_{\rho}(I)=Q_{\rho}\left(I_{0}\right) \exp \left(\left.f_{N}\right|_{I}\right)
$$

if $I \in \Lambda_{N}$, thus $Q_{\rho}(I) \sim e^{f_{l}}$ with a constant that depends only on $\lambda, K,\|\mu\|_{C}$. Let

$$
\mathscr{F}=\left\{I \in \Lambda:\left|\frac{\int_{I} f}{|I|}\right| \geq \frac{\left|f_{I_{0}}\right|}{\varepsilon_{0}}\right\}, \quad E=\bigcup_{I \in \mathscr{F}} I, \quad \text { and } \quad S=I_{0} \backslash E .
$$

We will choose $\varepsilon_{0}$ later on. As in Theorem 6.2 , we can use a stopping time argument to show that $|E| \leq \varepsilon_{0}\left|I_{0}\right|$. If $d \in S$ and $I \in \Lambda$ contains $d$ then

$$
\left|f_{I}\right| \leq\left|f_{I_{0}}\right| / \varepsilon_{0} \leq C\left(\lambda, K,\|\mu\|_{C}, \varepsilon_{0}\right) .
$$

Thus if $d \in S$ and $I \in \Lambda$ contains $d$, we have

$$
Q_{\rho}(I) \sim 1
$$

with a constant that depends only on $\lambda, K,\|\mu\|_{C}$, and $\varepsilon_{0}$. If $\mathscr{Z}=z(E)$ then $H_{1}(\mathscr{Z}) \leq 2 \varepsilon_{0}\left|I_{0}\right| \leq 4 \varepsilon_{0} H_{1}(\gamma)$, because $z$ is a 2-parameterisation of $\gamma$. 
Take $\mathscr{Z}_{n}$ to be the set $\mathscr{Z}$ obtained when $\gamma=\gamma_{n}$. Now let $\mathscr{E}=\bigcup_{n=1}^{N} \mathscr{Z}_{n}$. Then

$$
H_{1}(\mathscr{E}) \leq 4 \varepsilon_{0} \sum_{n=1}^{N} H_{1}\left(\gamma_{n}\right) \leq 4 \varepsilon_{0} C(\lambda) H_{1}\left(\Gamma_{0} \cap B\right) .
$$

Now choose $\varepsilon_{0}$ so that $4 \varepsilon_{0} C(\lambda)=\varepsilon$ and let $W=\left(\Gamma_{0} \cap B\right) \backslash \mathscr{E}$. Let $x, y \in W$. Then $x \in \gamma_{j}$ and $y \in \gamma_{k}$ for some $j, k$. Take $\gamma$ to be $\gamma_{j}$. If $d \in z^{-1}(x)$ then $d \in S$. As in the proof of (6.4) in Theorem 6.2 we can find $I=[a, b] \in \Lambda$ for which

$$
\frac{1}{A(\lambda, K)} \leq\left|\frac{\rho(x)-\rho(y)}{y-x}\right|\left|\frac{z(b)-z(a)}{\rho(z(b))-\rho(z(a))}\right| \leq A(\lambda, K) .
$$

Combining this with (6.9) we obtain

$$
\frac{1}{M\left(\lambda, K,\|\mu\|_{C}, \varepsilon\right)} \leq\left|\frac{\rho(x)-\rho(y)}{y-x}\right| \leq M\left(\lambda, K,\|\mu\|_{C}, \varepsilon\right) .
$$

Corollary 6.4 characterised the images of A-D curves when $\|\mu\|_{C}$ is small and Corollary 6.5 characterised the images of chord-arc curves when $\|\mu\|_{C}$ is small. Even when there is no restriction on the size of $\|\mu\|_{C}$ we can still characterise the images of chord-arc curves. The images are certainly quasicircles but the next theorem shows that they are also Bishop-Jones curves.

It is straightforward to check that if $\rho \in \Sigma_{C}(E)$ and $g=\rho \circ \tau^{-1}$, where $\tau$ is Möbius, then $\left\|\mu_{g}\right\|_{C}$ relative to $\tau(E)$ is bounded by a constant times $\left\|\mu_{\rho}\right\|_{C}$.

A curve $\Gamma_{0}$ is chord-arc if and only if it is chord-arc in the spherical metric. Therefore if we lift it onto the sphere in the usual way its image is a chord-arc curve on the sphere. The map $\tau(z)=z^{-1}$, when lifted to the sphere, an isometry, so $\tau\left(\Gamma_{0}\right)$ is chord-arc in the spherical metric and hence in the Euclidean metric. It follows that the image of $\Gamma_{0}$ under any Möbius transformation is also a chord-arc curve.

Theorem 6.7. If $\Gamma_{0}$ is an $\alpha$ chord-arc curve, and $\rho$ is a $K$ quasiconformal map whose dilatation $\mu$ satisfies $\|\mu\|_{C}<\infty$ relative to $\Gamma_{0}$, then $\rho\left(\Gamma_{0}\right)$ is a BJ curve with constants that depend only on $\alpha,\|\mu\|_{C}, K$.

Proof. $C, M$ will denote constants that depend at most on $\alpha,\|\mu\|_{C}, K$, and $A$ will denote any universal constant. Our first step will be to reduce to the case $\Gamma_{0}=\mathbf{R}$. Using Möbius transformations and the comments immediately preceding this theorem we can reduce to the case where $\Gamma_{0}$ goes through infinity and $\rho$ fixes infinity. By Lemma 4.11 in [S1] there is a $C(\alpha)$ bi-Lipschitz map $\tau$ of $\mathbf{C}$ that fixes $\infty$, maps $\mathbf{R}$ onto $\Gamma_{0}$ and for which $\left\|\mu_{\tau}\right\|_{C} \leq C(\alpha) \quad\left(\mu_{\tau}\right.$ is the dilatation of $\tau)$. Let $\rho_{1}=\rho \circ \tau$, then $\left\|\mu_{\rho_{1}}\right\|_{C} \leq C$ and $\rho_{1} \circ \tau(\mathbf{R})=\Gamma_{0}$. So we can assume that $\Gamma_{0}=\mathbf{R}$.

Let $\Omega$ be one of the components of $\mathbf{C} \backslash \rho(\mathbf{R})$ and $z_{0} \in \Omega$. Take $\psi$ to be the Riemann map of $\mathbf{R}_{+}^{2}$ onto $\Omega$ that fixes $\infty$ and maps $i$ to $z_{0}$. Because $\rho(\mathbf{R})$ is a $K$ quasicircle we can extend $\psi$ to a $C(K)$ quasiconformal map of C. We will build a chord-arc domain $\mathscr{D}$ containing $z_{0}$ such that $\psi(\mathscr{D})$ is a chord-arc domain.

Define $I_{0}=\rho^{-1}(\psi[-1,1])$. By Theorem 6.6 there is a set $W_{0} \subseteq I_{0}$ such that $\left|W_{0}\right| \geq 2^{-1}\left|I_{0}\right|$ and

$$
\left.\frac{\left|I_{0}\right|}{\operatorname{diam} \rho\left(I_{0}\right)} \rho\right|_{W_{0}}
$$


is $M$ bi-Lipschitz. We can assume that $W_{0}$ is closed, so we can write $I_{0} \backslash W_{0}=$ $\bigcup_{k \geq 1} I_{k}$, where $I_{k}=\left(a_{k}, b_{k}\right)$ and the $I_{k}$ are disjoint. Let $W=\psi^{-1}\left(\rho\left(W_{0}\right)\right)$ and $J_{k}=\left(s_{k}, t_{k}\right)=\psi^{-1}\left(\rho\left(I_{k}\right)\right)$. For each $J_{k}$ define $\sigma_{k}$ to be the top two edges of the equilateral triangle with base $J_{k}$ and $\sigma_{0}$ will be the top three sides of the square with base $[-1,1]$. Taking

$$
\Sigma=\sigma_{0} \cup\left(\bigcup_{k \geq 1} \sigma_{k}\right)
$$

it is easily seen that $\mathscr{D}=\operatorname{Int}(\Sigma)$ is an $A$ chord-arc domain.

It is shown in [JK] that if $L$ is a line segment in $\mathbf{R}_{+}^{2}$ that is orthogonal to $\mathbf{R}$ then $\psi(L)$ is chord-arc. The same proof shows that each $\psi\left(\sigma_{k}\right)$ is $C(K)$ chord-arc. Then $\Gamma_{1}=\psi(\Sigma)$ consists of chord-arc pieces. We will use Theorem 6.6 to show that the pieces join up to form a chord-arc curve.

Let $x, y \in W_{0}$ and $S=\left\{k: I_{k} \subseteq[x, y]\right\}$. Then

$$
[x, y]=\left([x, y] \cap W_{0}\right) \cup\left(\bigcup_{k \in S} I_{k}\right) .
$$

Because $\left.\rho\right|_{W_{0}}$ is multiple of an $M$ bi-Lipschitz function and $a_{k}, b_{k} \in W_{0}$ we can deduce that

$$
H_{1}\left(\rho\left([x, y] \cap W_{0}\right)\right)+\sum_{I_{k} \in S}\left|\rho\left(a_{k}\right)-\rho\left(b_{k}\right)\right| \leq M^{2}|\rho(x)-\rho(y)| .
$$

If $a, b \in W$ and $T=\left\{k: J_{k} \subseteq[a, b]\right\}$ we can rewrite (6.11) as

$$
H_{1}(\psi([a, b] \cap W))+\sum_{k \in T}\left|\psi\left(s_{k}\right)-\psi\left(t_{k}\right)\right| \leq M^{2}|\psi(a)-\psi(b)| .
$$

Because $\psi\left(\sigma_{k}\right)$ is chord-arc we have

$$
H_{1}(\psi([a, b] \cap W))+\sum_{k \in T} H_{1}\left(\psi\left(\sigma_{k}\right)\right) \leq C|\psi(a)-\psi(b)| .
$$

We want to check that $\Gamma_{1}=\psi(\varepsilon)$ is a chord-arc curve. Let $a, b$ be two points on $\Sigma$. There are four cases to consider:

Case (i) $a, b$ are both elements of some $\sigma_{j}$.

Case (ii) $a, b$ are both elements of $W$.

Case (iii) $a \in \sigma_{j}$ and $b \in W$.

Case (iv) $a \in \sigma_{j}$ and $b \in \sigma_{k}, j \neq k$.

Case (i) is done already because $\psi\left(\sigma_{j}\right)$ is chord-arc. Next we will prove case (ii). Let $\gamma$ be the path from $a$ to $b$ that goes along the base of $\Sigma$. Then

$$
\gamma=([a, b] \cap W) \cup\left(\bigcup_{k \in T} \sigma_{k}\right)
$$

and therefore it follows from (6.12) that

$$
H_{1}(\psi(\gamma)) \leq C|\psi(a)-\psi(b)| .
$$

This proves (ii). 
Now for (iii). There is $t \in \sigma_{j} \cap W$ such that $|t-a|,|t-b| \leq A|b-a|$ and because $\psi$ is $C(K)$ quasiconformal we also have

$$
|\psi(t)-\psi(a)|,|\psi(t)-\psi(b)| \leq C(K)|\psi(b)-\psi(a)| .
$$

Let $\gamma_{1}$ be the arc from $a$ to $t$ along $\sigma_{j}$ and let $\gamma_{2}$ be the arc from $t$ to $b$ along the base of $\Sigma$. Using case (i) for $\gamma_{1}$, case (ii) for $\gamma_{2}$, and (6.13) we get (iii).

The proof of case (iv) is similar.

So we have shown that $D=\operatorname{Int}\left(\Gamma_{1}\right)$ is a $C$ chord-arc domain. Using the quasiconformal distortion theorems we get that

$$
\operatorname{diam}\left(\rho\left(I_{0}\right)\right)=\operatorname{diam}(\psi([-1,1])) \sim d\left(z_{0}, \rho(\mathbf{R})\right) \sim \operatorname{diam}(\partial D) .
$$

Because $H_{1}(\partial D) \sim \operatorname{diam}(\partial D)$ we have $H_{1}(\partial D) \sim d\left(z_{0}, \rho(\mathbf{R})\right)$. The only remaining $\mathrm{BJ}$ condition that we have to check is that $H_{1}(\partial D \cap \rho(\mathbf{R}))$ is not too small. From (6.10) and (6.14) we get

$$
\begin{aligned}
H_{1}(\partial D \cap \rho(\mathbf{R})) & =H_{1}\left(\rho\left(W_{0}\right)\right) \geq \frac{\left|W_{0}\right|}{M\left|I_{0}\right|} \operatorname{diam}\left(\rho\left(I_{0}\right)\right) \\
& \geq \frac{1}{2 M} \operatorname{diam}\left(\rho\left(I_{0}\right)\right) \geq \frac{1}{C} d\left(z_{0}, \rho(\mathbf{R})\right) .
\end{aligned}
$$

It was mentioned in the introduction that this result can also be proven by combining several results from the literature. As in the proof above we can reduce to the case where $\Gamma_{0}$ is the real line. The next step is to replace $\rho$ by a quasiconformal map which is the same as $\rho$ on the real axis but is better behaved off the real line and whose dilatation still satisfies the Carleson condition. Let $h=\psi^{-1} \circ \rho$. Then by results in [FKP], or by Theorem 6.2(iii), $h$ is locally absolutely continuous on $\mathbf{R}$ with derivative in $A_{\infty}$. Taking $\tau$ to be the Beurling-Ahlfors extension of $h$ we define $H$ in the upper half-plane to be $\psi \circ \tau$. The restriction of $H$ to the real line is equal to $\rho$, and $H$ and $\tau$ have the same dilatations. $H$ is defined similarly in the lower half-plane. It is easily checked that

$$
\frac{1}{C} \leq \frac{y|\partial \tau|}{\operatorname{Im} \tau} \leq C
$$

Because $h$ is in $A_{\infty}$ we also get that the dilatation of $\tau$ satisfies the Carleson condition.

Now let $\rho_{1}$ be the mapping which is symmetric about $\mathbf{R}$ and is equal to $\tau$ in the upper half-plane. Then $H$ can be written as $\rho_{2} \circ \rho_{1}$ where $\rho_{2}$ is conformal in the upper half-plane. Having the derivative of $\left.\tau\right|_{\mathbf{R}}$ in $A_{\infty}$ along with (6.15) means that the conditions of Lemma 4.8 in [S1] are satisfied and so $\rho_{1}$ preserves Carleson measures. This ensures that the dilatation of $\rho_{2}$ satisfies the Carleson condition. Now we can apply the result $(0.1)$ of the introduction to $\rho_{2}$ to deduce that the image of $\mathbf{R}$ is a B-J curve.

Theorem 6.7 has the following converse:

If $\Omega$ is a simply connected domain whose boundary $\Gamma$ is a quasicircle and a $B J$ curve and if $\psi$ is a Riemann map from $\mathbf{R}_{+}^{2}$ to $\Omega$ then $\psi$ can be extended to a quasiconformal map of $\mathbf{C}$ whose dilatation $\mu$ satisfies $\|\mu\|_{C}<\infty$ relative to $\mathbf{R}$. 
This is one of the parts of Theorem 4 in [AZ]. They used the unit disc instead of $\mathbf{R}_{+}^{2}$ but this does not matter because the case of $\mathbf{R}_{+}^{2}$ can be deduced from the case of the unit disc.

The final theorem that we will prove is Theorem 1 of [AZ]. This theorem allows us to extend Theorem 6.2 in the case where $\Gamma_{0}=\mathbf{T}$, by allowing $\rho$ to be conformal in $\mathbf{D}$.

Theorem 6.8. Let $\rho$ be a quasiconformal map that is conformal in $\mathbf{D}$ and whose dilatation $\mu$ satisfies $\|\mu\|_{C}<\infty$ relative to $\mathbf{T}$. Then $\log \rho^{\prime} \in \operatorname{BMOA}(\mathbf{D})$.

Proof. $\operatorname{BMOA}(\mathbf{D})$ is those functions that are in $H^{2}(\mathbf{D})$ with boundary values in $\operatorname{BMO}(\mathbf{T})$. We will initially assume that $\rho$ fixes $\infty$.

Let $\Lambda_{k}$ be the division of $\mathbf{T}$ into $2^{k}$ pieces of length $2 \pi 2^{-k}$ and let $\Lambda=$ $\bigcup_{k \geq 0} \Lambda_{k}$. This is not quite the good subdivision constructed in Chapter 2 . There we parameterised $\mathbf{T}$ by an interval but here we are parameterising $\mathbf{T}$ by itself. Proposition 4.1 is true with this subdivision.

For $I \in \Lambda$ let $r I=\left\{r e^{i \theta}: e^{i \theta} \in I\right\}$ and $r \Lambda_{k}=\left\{r I: I \in \Lambda_{k}\right\}$. Then $\left(1-2^{-k}\right) \Lambda_{k}$ divides $\left(1-2^{-k}\right) \mathbf{T}$ into $2^{k}$ equal intervals. From the quasiconformal distortion theorems and the Koebe 1/4 Theorem we get that

$$
\left|\rho^{\prime}(x)\right| /\left|Q_{\rho}(I)\right| \sim 1 \text { for } I \in \Lambda_{k}, x \in\left(1-2^{-k}\right) I .
$$

Combining this with Proposition 4.1 we see that

$$
\left|\rho^{\prime}(x)\right| /\left|Q_{\rho}\left(I_{0}\right)\right| \sim\left|e^{f_{I}}\right| \text { for } I \in \Lambda_{k}, x \in\left(1-2^{-k}\right) I .
$$

It follows that

$$
\int_{\left(1-2^{-k}\right) \mathbf{T}}|\log | \rho^{\prime}(x)|-| Q_{\rho}\left(I_{0}\right)||^{2} \leq C+\|f\|_{2}^{2}<\infty .
$$

This is true for all $k$, thus $\log \left|\rho^{\prime}\right|$ is the Poisson integral of its boundary values $\operatorname{Re} f$, which is in $\operatorname{BMO}(\mathbf{T})$. Therefore $\operatorname{Im}\left(\log \rho^{\prime}\right)$, is the Poisson integral of a $\operatorname{BMO}(\mathbf{T})$ function (see Chapter VI, [G]). Thus $\log \rho^{\prime} \in \operatorname{BMOA}(\mathbf{D})$ when $\rho$ fixes $\infty$.

Now assume that $\rho$ does not fix $\infty$. We can assume that $\rho(\infty)=0$. Let $\tau(z)=z^{-1}$. Then $\rho=\tau \circ G$ where $G$ is one of the functions that we have already proven the result for. Because

$$
\log \rho^{\prime}(x)=-2 \log G(z)+\log G^{\prime}(z)
$$

we only have to check that $\log G(z) \in \operatorname{BMOA}(\mathbf{D})$. This can be done using the quasiconformal distortion estimates to get a John-Nirenberg type estimate for $\log |G(z)|$ on any $r I$, and then proceeding as above.

\section{REFERENCES}

[A] L. V. Ahlfors, Lectures on quasiconformal mappings, Wadsworth and Brooks/Cole, Monterey, Calif., 1987.

[AZ] K. Astala and M. Zinsmeister, Teichmüller spaces and BMOA, preprint, 1990.

[BJ] C. Bishop and P. W. Jones, Harmonic measure, $L^{2}$ estimates and the Schwarzian derivative, J. Analyse Math. (to appear).

[C] L. Carleson, On mappings, conformal at the boundary, J. Analyse Math. 19 (1967), 1-13.

[D] B. Dahlberg, On the absolute continuity of elliptic measures, Amer. J. Math. 108 (1986), $1119-1138$. 
[F] K. J. Falconer, The geometry of fractal sets, Cambridge Tracts in Math., vol. 85, Cambridge Univ. Press, Cambridge, 1985.

[FKP] R. Fefferman, C. Kenig, and J. Pipher, The theory of weights and the Dirichlet problem for elliptic equations, Ann. of Math. (2) 134 (1991), 65-124.

[J] P. W. Jones, Lipschitz and bi-Lipschitz functions, Rev. Mat. Iberoamericana 4 (1988), 115121 .

[JK] D. S. Jerison and C. E. Kening, Hardy spaces, $A_{\infty}$ and singular integrals on chord-arc domains, Math. Scand. 50 (1982), 221-248.

[L] O. Lehto, Univalent functions and Teichmüller spaces, Graduate Texts in Math., SpringerVerlag, New York and Berlin, 1987.

[LV] O. Lehto and K. Virtanen, Quasiconformal mappings in the plane, Springer-Verlag, Berlin and New York, 1973.

[S1] S. Semmes, Quasiconformal mappings and chord-arc curves, Trans. Amer. Math. Soc. 306 (1988), 233-263.

[S2] Estimates for $(\bar{\partial}-\mu \partial)^{-1}$ and Calderón's theorem on the Cauchy integral, Trans. Amer. Math. Soc. 306 (1988), 191-232.

[St] E. Stein, Singular integrals and differentiability properties of functions, Princeton Univ. Press, Princeton, N.J., 1970.

Department of Mathematics, Yale University, New Haven, Connecticut 06520 78712

Current address: Department of Mathematics, University of Texas at Austin, Austin, Texas

E-mail address: pmm@math.utexas.edu 